(Aus dem physiologischen Institut zu Königsberg i. Pr.)

\title{
Weitere Untersuchungen über das Wesen der Vocale.
}

(Unter Mitwirkung: des Herrn H. H i r s h f eld.)

Von

L. Hermanu.

(Hierzu Tafel V und VI.)

1. Vorbemerkung und Methodisches.

Die fortschreitende Vervollkommnung meines Verfahrens, die Eindrücke des Edison'schen Phonographen auf photographischem Wege in Curven umzusetzen, liess es wünschenswerth erscheinen, die Curven der 9 Hauptvocale in möglichst vollkommener Form zu gewinnen, um sie durch trene Reproduction jedem, der sich für den Gegenstand interessirt und selbstständig Messungen 7.u machen wïnscht, zugänglich zu machen: Ausserdem lag mir daran, auch von den kurzen Vocalen (wie in Ann, Enn), welche ich bisher nur gelegentlich berührt habe ${ }^{1}$ ), vollständige Analysen zu gewinnen.

Ich habe daher in den letzten Monaten eine grössere Anzahl Blätter ausschliesslich für diesen Zweck angefertigt. Erfahrungsgemäss sind sehr grosse Curven für genaue Ausmessung weniger bequem, als solche, deren Messung auf dem von mir beschriebenen Wege ${ }^{2}$ ) unter dem Microscop micrometrisch erfólgen kann. Der vor einigen Jabren von R. Fuess in Steglitz bei Berlin für das Institut angefertigte vorzügliche micrometrische Messtisch gestattet ohne Hilfslinien Curven zu messen, deren Abscissenlänge nicht uber $36 \mathrm{~mm}$, und deren Ordinatenlängen nicht über $16 \mathrm{~mm}$ gehen; grössere

1) Dies Archiv Bd. 58. S. 260.

2) Dies Archiv Bd. 47. S. 46. Ueber Ausmessung grösserer Curven s. Bd. 58, S. 265. 
Dimensionen köunen noch gemessen werden, wenn eine mit einer feinen Nadel auf das Papier eingekratzte Hilfsaxe und nöthigenfalls auch eine Hilfsordinate benutzt wird. Der Objecttisch besitzt eine Micrometerschraube, deren Knopftheilung Vierhundertstel mm direct abzulesen gestattet. Diese Schraube wird benutzt, um die Periodenlänge zu messen und die Curve, welche zwischen 2 Glasplatten befestigt ist, genau um Vierzigstel der Periodenlänge zu verschieben. In derselben Richtnng wirkt auch eine Triebbewegung verschiebend, deren Theilung mit Nonius Zehntel mm abzulesen erlaubt. Diese Bewegung dient zur Ergänzung der Weglänge der Schraubenbewegung. Die Ordinatenmessung erfolgt mittels einer zur Axenrichtung senkrechten Triebbewegung, deren Theilung und Nonius auf Zehntel mm genau ist. Die Messungen erfolgen mit einem Fadenkreuz.

Das Institut besitzt zwei Accumulatoren, der eine von 6, der andere von 30 Elementen, beide im Souterrain aufgestellt, und durch ein 3-Leiter-System unabhängig von einander in den verschiedenen Institutsräumen benutzbar. Der kleinere Accumulator dient zur Bewegung des Phonographen beim Abdrehen, Besingen und Abhören, der grössere zum Betrieb der Bogenlampe für die Curvenaufnahmen. Bei den letzteren wird der Phonograph mittels eines Eichens'schen Uhrwerkes äusserst langsam gedreht, mit einer Geschwindigkeit, welche nur $1 / 300$ bis $1 / 450$ der zum Besingen verwendeten ist.

Zur Ueberführung der Eindruicke in Bewegungen des photographirenden Lichtstrahls stebt eine stärkere und eine schwächere Hebelvergrösserung zur Verfügung, die erstere mit 3, die letztere mit nur 2 Hebelaxen. Die beiden, allmählich sehr verbesserten Apparate sind schon früher schematisch abgebildet, der erstere Bd. 58. Tafel II. Fig. 1, der zweite Bd. 55. Tafel I. Fig. 5 und 6. Für Vocalcurven von zur microscopischen Messung bequemen Dimensionen wird stets der letztere Apparat benutzt. Ein anderes Mittel zur Beherrschung der Ordinatenhöhen ist die Länge des reflectirten Lichtstrahls, d. b. der Abstand des Spaltbildes vom Spiegelchen. Diese Länge, und mit ihr die Ordinatengrösse, ist um so geringer: 1. je weiter der an der electrischen Lampe angebrachte belenchtete Spalt vom Phonographen entfernt ist, 2. je stärker die vor dem Spiegelapparat angebrachte Convexlinse (1 bis $1 \frac{1}{2}$ Dioptrien). 
Die Periodenlängen der Curven sind bei gegebener Tonhöhe um so grösser: 1. je langsamer die Reproductivdrehung des Phonographencylinders im Verhältniss zur Drehung beim Besingen, 2. je 'schneller die Drehung des Baltzar'schen Cylinders, auf dem das Bromsilberpapier befestigt ist.

Aus vorstebenden Bemerkungen ergeben sich die Mittel, welehe zur Verfügung stehen, um Curven von gewünschten Diménsionen zu erhalten.

Für die Vocalcurven der neuen Serie wurde auf selbstthätige Axenverschiebung des Baltzar'schen Cylinders verzichtet, hauptsächlich damit die Abscissenaxen der Curven den Papierrändern genau parallel verlaufen. Nach jedem Umgange des Cylinders wird derselbe mittels der Kurbel soweit verschoben, wie nöthig ist, damit sich die Curven nirgends ïberdecken.

Um den Cylinder nach jedem Umgange, oder nach jedem halben oder viertel Umgange, sicher zum Stehen zu bringen, was sehr wichtig ist, habe ich folgende einfache Vorrichtung angebracht. Der bekannte Baltza r' sche Cylinder hat an seinen beiden Grundflächen je 4 platte Speichen. In jede der Speichen derjenigen Grundfläche, welche der Verschiebungskurbel zunächst steht, und dicht am Verschiebungsarm vorbeigeht, wurde ein feines Loch gebohrt, in welche ein kurzer geknöpfter Vorstecker hineinpasst. Mittels dieses Vorsteckers, welcher beim Rotiren des Cylinders schliesslich sich am Verschiebungsarm fängt, kann man dem $\mathrm{Cy}$ linder nach Belieben ganze, halbe oder viertel Umdrehungen freigeben. Das Umschalten des Vorsteckers sowie die Kurbeldrehungen erfolgen nach Auslöschen des electrischen Lichtes und Kleinstellen der Gaslampe unter Lüften des die Schutzkammer bedeckenden Sammettuches.

Das ganze Verfahren ist in allen seinen Theilen so sicher, dass ein Missrathen eines Blattes schon seit langer Zeit nicht mebr vorkommt.

2. Die Anaperiodicität der unharmonischen Formanten.

Die erste Aufgabe, welche ich mir stellte, war die Untersuchung, ob die unhạrmonischen Bestandtheile der Vocalklänge sich in jeder Periode unabhängig einsetzend wiederholen, oder ob sie eine in der Phase fortlaufende und nur mit jeder Stimmperiode unterbrochene Schwingung darstellen. Im letzteren Falle 
müsste die unharmonische Schwingung sich gegen dieStimmperioden noniusartig: versebieben. Der Kür\%e halber werde ich im folgenden den ersten Fall als einen an aperiodiscben, den zweiten als einen a toperiod is shen unharmonischen Bestandtheil bezeichnen: Von Anfang an habe ich es aus dem blossen Anblick der Curven für wahrscheinlicher gehalten, dass die unharmonisehen Formanten anaperiodisch auftreten 1), aber wiederholt darauf hingewiesen, dass dies nur durch Analyse mehrerer Perioden derselben Curven entschieden werden $\mathrm{kann}^{2}$ ). Allerdings zeigen längere Vocalcurven nicht selten kleine Verschiedenheiten im Anssehen der einzelnen Perioden, und diese Verschiedenheiten stellen sich allmäblich in einer gewissen Entwicklungsreihe ein. Aber die Ursache hiervon braucht nicht in autoperiodischer Disliarmonie, sondern kann ebensogut in leichtem Detoniren der Stimmnote liegen. Die Analyse muss jedenfalls hierüber Aufschluss geben. Ist nämlich der Formant autoperiodisch, so miissen sich die hervorragenden Partialtöne in der Phase regelmässig verschieben, ist er dagegen anaperiodisch, so wird dies nicht der Fall sein, anch wenn die Stimme detonirt.

Unterdessen bat Pipping in einer neueren Arbeit ${ }^{3}$ ), auf welche ich unten zurückkomme, dieselbe Frage bearbeitet, wenn auch, da er keine unharmonischen Formanten zugiebt, von etwas anderem Gesichtspunkte. Er ist zu dem Resultat gelangt, dass keine Phasenverschiebungen vorkommen, welche nicht auf Detoniren des Stimmklanges bezogen werden könnten.

Die. auf die vorliegende Frage beziiglichen Messungen und Analysen sind auf meine Veranlassung von Herrn H. Hirschfeld vorgenommen worden. $\mathrm{Zu}$ denselben wurden Curven verwendet, welche sich über die ganze, $50 \mathrm{~cm}$ betragende Länge des Papierblattes erstreckten, und je nach der Tonhöhe 26 bis 52 Perioden enthielten. Es wurden natïrlich nur solche Curven verwendet, welche die oben angedenteten leichten Variationen der Periodenformen zeigten, und es wurden solche Perioden analysirt, in welchen diese Verschiedenheiten ihr Maximum erreicht hatten. Gewöhnlich wurden nur 10 Partialschwingungen berechnet, auch wo die restiren-

1) Vgl. dies Archiv Bd. 47. S. 381; Bd. 48. S. 183 f., 187 ; Bd. 58. S. 275.

2) Dies Archiv Bd. 48. S. 187, Anm.; Bd. 58. s. 275.

3) Zeitschr. f. Biologie Bd. 31. S. 524. Genaueres in Acta societ. scient. Fenuicae Bd. 20. No. 11. Helsingfors 1894. 
den Glieder noch ziemlichen Betrag hatten, da es hier eben nicht auf vollständige Analysen ankam. Ich theile im Folgenden einige Beispiele mit. Auch wo mehr als 10 Glieder berechnet worden sind, setze ich hier nur die ersten 10 her. Alle Amplituden sind auf gleiche Curvenböhen, nämlich $10 \mathrm{~mm}$ reducirt, und bedeuten Zehntel$\mathrm{mm}$, also Procente der maximalen Curvenhöhe zwischen den untersten und den obersten Culminationen.

Die Phasen sind so umgerechnet, dass der hervorragendste Partialton mit der Phase 0 einsetzt, und auf volle Grade abgerundet.

\section{Vocal $A$, Note $e$. Táfel 270.}

Amplituden.

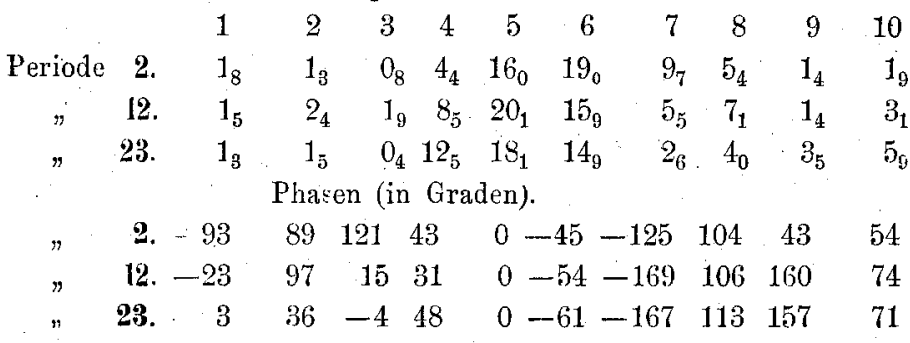

Vocal $A$, Note $e$. Tafel 271.

Amplituden.

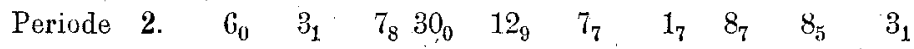
$\begin{array}{lllllllllllll} & 30 . & 4_{1} & 4_{6} & 3_{7} & 30_{1} & 13_{8} & 10_{8} & 3_{6} & 8_{5} & 7_{4} & 4_{4}\end{array}$ Phasen.

2. $-143-10 \quad 50 \quad 0-61 \quad 113+180 \quad 81 \quad 1-153$

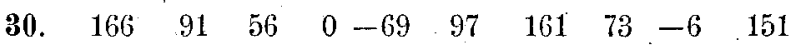

Vocal $A$, Note $c^{1}$. Tafel 271.

Amplituden.

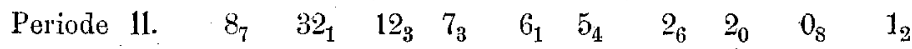

$\begin{array}{llllllllllll} & 37 . & 8_{1} & 38_{9} & 18_{0} & 6_{4} & 6_{2} & 4_{1} & 2_{9} & 1_{2} & 1_{5} & 0_{4}\end{array}$ Phasen.

11. $\quad 109 \cdot 0 .-169 \quad 44-97 \quad 64-86 \quad 125 \quad-64 \quad 172$

37. $113 \quad 0-174-54 \quad 121 \quad 20 \quad 174 \quad 53 \quad 109 \quad 28$

Vocal $O$, Note $c$. Tafel 269 .

A mplituden.

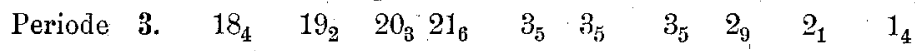
$\begin{array}{lllllllllll}\text { "20. } & 12_{9} & 22_{9} & 18_{1} & 22_{7} & 18_{4} & 5_{6} & 5_{2} & 2_{8} & 5_{3} & 1_{6}\end{array}$ Phasen.

" 3. $-86-162 \quad 153 \quad 0 \quad 120-82-160 \quad 16 \quad 124-104$

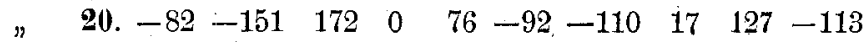


Wie man sieht, sind die Analysen mehrerer Perioden desselben Vocalklanges, trotz des etwas verschiedenen Aussehens derselben, sowohl in den Amplituden wie in den Phasen ziemlich übereinstimmend. Wo grosse Abweichungen vorkommen, betreffen dieselben Partialtöne, welche wenig hervorragen. Jedenfalls ist der Schluss gerechtfertigt, dass eine regelmässige Verschiebung, wie sie ein autoperiodischer unharmoniseher Formant verursachen wïrde, nicht vorkommt, so dass also die von mir nachgewiesenen unharmonischen Bestandtheile als anaperiodisch zu betrachten sind.

Unter den zahlreichen, noch zu beleuchtenden Einwänden, welche H. Pipping neuerdings gegen meine Vocaluntersuchungen erhoben hat ${ }^{1}$, figurirt auch von Neuem der schon von ihm und Hensen gemachte, und trotz meiner Zurïckweisung ${ }^{2}$ ) von $A$ uerbach wiederholte, dass die von mir behaupteten unharmonischen Formanten beim vocalisirten Gesang jeden Kunstgenuss unmöglich machen würden. Da Pipping jetzt selber einsieht, dass dieser Einwand nur dann zutreffend sein würde, wenn die unharmonischen Formanten autoperiodisch wären (S. 529) $)^{3}$ ), so kann ich mir seinen Einwand nur aus dem Missverständniss erklären, dass ich die letztere Ansicht ausgesprochen hätte. Bei aufmerksamem Lesen wiirde er gefunden haben, dass ich stets nur anaperiodische Disharmonie behauptet, und nur die Möglicbkeit autoperiodiseher durch besondere Untersuchung ausgeschlossen zu sehen gewünscht babe. Der Einwand fällt also in Nichts zusammen.

3. Neue Curven langer Vocale (Tafel V) und Principien der Untersuchung.

$\mathrm{Zu}$ dem eingangs angegebenen $\mathrm{Zwecke}$ gebe ich auf Taf. $\mathrm{V}$ eine Anzahl möglichst treuer Copien von Vocalcurven in denjenigen Dimensionen, wie sie in der letzten Zeit hauptsächlich za unseren Messungen gedient haben. Sehr gern hätte ich photographische Reproductionen gegeben, aber die Originalcurven erwiesen sich

1) Zeitschr. f. Biologie Bd. 31. S. 524.

2) Dies Archiv Bd. 48. S. 184.

3) Anstatt der Worte: "Es frent mich, wenigstens in diesem Puncte Prof. H e r m a n beistimmen zu können" hätte der Verf. besser gesagt: „es drängt mich meinen irrigen Angriff zurückzunehmen." 
dazu im Allgemeinen als zu fein; es musste daher wieder zum Pausverfabren gegriffen werden. Von der Glätte und Eleganz der Originale geben die Reproductionen keine Vorstellung, jedoch sind sie bis auf. einige Härten in der Wiedergabe feiner Details so correct, dass sie zur Analyse benutzt werden können.

Aus den meist 50 oder $25 \mathrm{~cm}$ langen Originalcurven (s. oben S. 171) sind nur Stücke von $8 \mathrm{~cm}$ wiedergegeben; nur 3 Curven sind fast in ganzer Länge des Originals ( 45 von $50 \mathrm{~cm}$ ) reprodacirt, um eine Vorstellung davon zu geben, wie die Perioden allmählich in Einzelheiten sich verändern. Auch zu den kurzen Curvenstücken sind, wo Veränderungen im Verlaufe der Curve bemerkbar waren, mit Vorliebe solche Strecken ausgewählt, in welchen die Veränderung sichtbar ist.

Durchweg ist links Vocal and Note, rechts in Haarschrift und eingeklammert die Blatt-Nummer angegeben. $\bar{A}, g$ bedeutet: langes $A$ auf Note $g$ gesungen. Die Stimmung ist die sog. mathematische, d. h. $c=128, e=160, g=192, c^{1}=256$ ganze Schwingungen p. Sec. Von einigen Vocalen habe ich mebrere Beispiele für dieselbe Note aus bestimmten Gründen darstellen lassen. Alle Curven rihhren von meiner Stimme her, für welche, wie man sieht, die Kraft einer Reihe von Vocalen $(E, I, A e, O e, U e)$ deutlich von $c$ bis $c^{1}$ zunimmt (dasselbe gilt für die entsprechenden kurzen Vocale, s. Taf. VI).

Die Wiederholung einer anaperiodisch unharmonischen Schwingung in jeder Periode springt besonders bei den Vocalen $A$ und $A o$ jedem Unbefangenen sofort in die Angen. Dass es sich hier nicht um willkürliche Auffassung handelt, ergiebt sich besonders beim Vergleich der Carven Nr. 1, 4, 5, 6, welche sämmtlich Vocal $\bar{A}$ auf Note $c$ darstellen und verschiedenen Blättern entnommen sind. In 5 sind jedesmal nur 2, in 1, 4, 6 dagegen 3 ganze Schwingungen des Formanten in jeder Periode enthalten; der Rest der Periode ist nahezu schwingungslos. Deutlicher kann die Selbstständigkeit des Formanten gegenüber der alten Lehre von der Verstärkung harmonischer Stimmpartialtöne gewiss nicht bewiesen werden. Auch Nr. 3 und 10 ( $A$ o, Note $c$ ) zeigen sehr schön eine selbstständige schwebungsartig unterbrochene und zugleich evident unharmonische, aber anaperiodische Formantschwingung. Dass der unmittelbare Anblick der Curven hier Dinge lehrt, welche in der 
Analyse niemals zu Tage treten können, wird Jeder zugeben, der nicht in theoretischer Voreingenommenheit befangen ist.

Den letzteren Vorwurf muss ich Pipping machen, dessen sonst schätzenswerthe und fleissige Untersuchung (Citat s. oben). durch diese Voreingenommenheit unfruchtbar wird. Er macht mir die seltsame Vorhaltung, dass ich die Curven anch mit dem Auge betrachte, während ,unsere Gesichtseindriicke für die Gehörwahrnehmung in keiner Weise massgebend sind", und dass ich „bei der Beurtheilung der Klangcurven die Empfindungen des Auges und die des Obres nicht streng auseinander balte". Als ob die Klangcurve etwas anderes wäre, als eine optische Darstellung der Schallbewegung, eben dazu gemacht, um mit allen Hilfsmitteln des Gesicbtssinnes studirt zu werden!. Zunächst kommt es darauf an, festzustellen, welche Art von objectiver Beweg ung der Vocalklang ist. Ein zweites, selbstständiges Problem ist es, wie das Gehororgan auf diesen Vorgang reagirt, eine Frage, auf welche ich weiter unten eingehe. Pipping betrachtet die Vocalfrage nur vom Standpunkt des Ohres, und noch dazu rom Standpunkt einer Hyp othese, die er fälschlich als Gesetz bezeichnet (s. unten S. 201), obwohl er sie, wie wir sehen werden, selber. in einem wesentlichen Punkte umzustossen sucht. Was zu dieser Hypothese nicht passt, ist er sehr geneigt, einfach als falsch anzusehen.

Ich kann nur wiederholen 1): die Curve ist der vollkommenste objective Ausdruck der vorliegenden Bewegung; wie wir die Curve lesen, ist willkürlich; die Auflösung in harmonische Theilschwingungen ist eine der Arten sie zu lesen, aber nicht die einzige.

Pipping freilich behauptet stets von Neuem, dass sie die einzige sei, obwohl er zu meiner Freude von einer ganzen Reihe schwerer Irrthümer seines erstẹn eiligen Angriffes in Folge meiner Beleuchtung desselben zurückgekommen ist. Er macht mir nicht mehr: wie friiher ${ }^{2}$ ) den Einwand, dass seine Analysen die Abwesenheit unharmonischer Formanten ergeben, weil die Curven sich ohne Fehler in harmonische Reihen auflösen lassen ${ }^{3}$ ), ja er hat sogar

1) Vgl. dies Archiv Bd. 58. S. 273. Diese am 16. October 1894 erschionene Arbeit war P i p p in $g$ bei der Abfassung seines Angriffs noch unbekannt. Ich bin genöthigt, einiges, dort schon Gesagte kurz zu reproduciren.

2) Zeitschr. f. Biologie Bd. 27. S. 433.

3) Noch immer ist übrigens Pipp ing über die Bedeutung seiner 
den von mir hervorgehobenen Umstand, dass diese Zerlegung eine willk uirliche ist'1), jetzt endlich auch aus Helmboltz herausgelesen. Er macht mir auch nicht mehr den Einwand, dass meine unharmonischen Formanten mit Resonatoren nicht nachweisbar sind, sondern gesteht zu (S. $531 \mathrm{Anm}$.), dass ich Recht batte diesen Nachweis bei anaperiodischen Formanten für unmöglich zu erklären ${ }^{-2}$. Ich hätte nur gewïnscht, dass ein Autor, der mich zum zweiten Male angreift, die Irrthümer seines ersten Angriffes ausdrücklich widerrufen bätte; der Leser merkt diese Widerrufe aus der Darstellung nicht.

Der jetzige Vorwurf Pipping's gegen meine Aufstellung anaperiodisch unharmonischer Formanten, dass nämlich diese Aufstellung auf einer anderen Betrachtungsweise a]s der analytischen beruht, ist also gar kein Einwand, da die analytische Betrachtung -diese Frage niemals entscheiden kann.

Ein zweiter Einwand gegen meine "Lehre" ist nach Pip ping. der Widerspruch zwischen derselben „und den bekanntesten Thatsachen der Acustik". Was meint wohl hier der Vf.? Dass ein Schall, wie ich ihn annehme, physikalisch undenkbar sei, wird doch selbst Pipping nicht behaupten wollen. Dass ein solcher, wie Pipping auf Grund eines unten zu besprechenden Versuches von Hensen irrthïmlich sehliesst, 'nicht auf die Weise zu Stande kommen könnte, wie ich es als möglich hingestellt habe, wiirde, wenn es wahr wäre, ebenfalls kein. Einwand gegen meine objective Anffassung der Vocalcurven und ebensowenig ein Widersprttch gegen bekannte Thatsacben der Acustik sein. Pi p ping sollte überhaupt so starke Ausdrücke auf einem Gebiete, das mir wohl ebenso bekannt ist wie ihm, nicht ohne die sorgfältigste Prïfung gebrauchen. Wie mir scheint, versteht Pip ing unter den „bekanntesten That-

Fehlerrechnung nicht völlig im Klaren; denn er sagt (S. 550), er habe mit derselben die Genanigkeit seiner "Messungen" viel sorgfältiger gepiüft, als irgend ein Anderer. Die $\mathrm{M}$ ess ung en werden aber durch die Fehlẹrrechnung absolut $\mathrm{n}$ ich $\mathrm{t}$ controlirt, sondern nur erstens die Richtigkeit der analytischen $\mathrm{R}$ e chn ung, zweitens, wieweit die berechneten Glieder ausreichen, die durch die gemessenen Ordinaten, mögen dieselben richtig oder falsch sein, gegebene Curve erschöpfend in Partialschwingungen auszudrücken.

1) Dies Archiv Bd. 48. S. 185.

2) Bd. 48. S. 183. 
sachen der.Acustik * wieder nar die.Hörhypothesen, in die er sich eingelebt lat, und die er mit Thatsachen verwechselt.

Ein dritter und neuer Einwand Pipping's gegen meine unharmonischen Formanten ist der allbekannte Helmholt $z^{\prime}$ sche Klavierversueh. Da anaperiodisch unharmonische Formanten die ibrem Ton entsprechende Saite nicht in Mitschwingung versetzen können, so könnten, wie Pipping meint, die Vocale, wie ich sie auffasse, nicht aus dem Klavier nachhallen, wäbrend sie es doch thun. Glaubt Pipping wirklich, dass ich einen so naheliegenden und (wenn er zutreffend wäre) schlagenden Einwand mir nicht selbst längst gemacht haben witrde? In der That habe ich schon vor 5 Jahren, zur Zeit meiner ersten Vocalarbeit, den Klavierversuch, den ich jedes Jahr meinen Zuhörern vorführe, und der auch mir im ersten Moment mit meiner Auffassung unvereinbar schien, sorgfältig elwogen, aber bei näherer Ueberlegung sofort. gefunden, dass dies kein Einwand ist. Und Pipping hätte ebenfalls, ehe er mit einem so wohlfeilen Einwand mich widerlegen zu können glaubte, die Sache genauer überlegen sollen. Sie ist nämlich ganz einfach. So gut wie sich jede Periode, auch mit einem anaperiodischen unharmonischen Formanten, in eine Fou rier'sche Reihe entwickeln lässt - und ich selbst habe in meinen Arbeiten viele solche Entwicklungen mitgetheilt ${ }^{1}$ ) -, so muss auch ein solcher Schall in einem möglichst vollständigen Resonatorensystem seine Vertretung finden, also auch sich durch Nachhall reproduciren. Ob der Formant selbst eine Saite ansprechen kann, wie es der Fall ist, wenn er mit einem Partialton des Stimmklanges zusammenfällt, oder ob dies, wie gewöhnlich, nicht der Fall ist, ist für den Erfolg völlig gleichgïltig. Auch dieser Einwand Pip ping's fällt also in Nichts zusammen. Auf den Klavierversuch komme ich unten nochmals zurtick.

Pipping's neuere Analysen ${ }^{2}$, welche an den angegebenen

1) Dies Archiv Bd. 48. S. 186, Anm; Bd.53. S. 46 ff.; Bd. 58. S. 276 ff.

2.) Die in diesen Analysen steckende Arbeit unterschätze ich keines. wegs, zumal Pip p ing, da er Nichts davon erwähnt, es offenbar verschmäht hat, sich des von mir erfundenen so ungemein bequemen Mittels der Schablonen (Bd. 47. S. 51) zu bedienen. Diese Schablonen lassen sich natürlich ebensoleicht für 48 , wie für meine 40 Ordinaten anfertigen. Pip ping hat sich sehr viel vermeidbare Arbeit gemacht. - Eine weitere Genauigkeitsp robe 
Stellen mitgetheilt sind, enthalten mehrere erfreuliche Annäherungen an meine Ergebnisse, obwobl bei der Verschiedenheit unserer Sprachen genug Differenzen unvermeidlich bleiben werden. Vor Allem findet er jetzt für $A$ ein früher nicht hervorgetretenes unteres Verstärkungsgebiet in der Gegend von gis ${ }^{2}$, also sehr nahe meinem Befunde. Für Ae geht die Uebereinstimmung noch weiter. Anch für $U$ ist die Uebereinstimmung grösser als ich früber meinte, da der von Pipping früher gefundene untere Formant bei $c^{1}$, und nicht, wie ich in Folge eines Druckfehlers bei ihm meinte, bei $c$ lag, bei mir bei $c^{1}$ bis $f^{1}$, und in Pipping's neuerer Angabe bei $d^{1}$ bis $f^{1}$. Den oberen Formanten von $U$, dessen "Entdeckung“" sich Pipping zuschreibt, und den ich gleichzeitig mit ihm ,entdeckt" babe, finde ich bei $d^{2}-e^{2}$, Pip ping legte inn früher bei $a^{2}$, jetzt bei $d^{3}$ (als Mittellagen) ${ }^{1}$ ). Gross sind und bleiben die Abweichungen besonders bei $O, O e, U e$ und $I$, deren tiefere von P ippin'g angegebene Formanten in meinen Analysen nicht auftreten. Dies bängt möglicherweise mit dem Umstande zusammen, dass in Pipping's Analysen im Allgemeinen die GrundtonAmplituden stärker hervortreten als in den meinigen, woraus Annahmen tiefer Formanten hervorgegangen sein können, zu welchen ich keine Veranlassung habe. In Pipping's älteren Analysen war allerdings das Hervortreten der Grundtöne noch viel stärker, wie folgende Zusammenstellung zeigt:

meines Scbablonenverfahrens (vgl. auch Bd. 53. S. 44) hat Herr Hirschfeld auf meine Veranlassung ausgeführt. Es wurden nämlich in der Productentabelle eines Analysenbogens die 4 obersten Reihen weggenommen und unten angehängt, d. h. der Nullpunkt der. Periode in der Rechnung um $36^{\circ}$ verschoben. Die nunmehrige Bestimmung der Amplituden stimmte ausgezeichnet zu der ersten; die Phasen aber waren natürlich gänzlich verändert, und zwar betrug, wie es sein muss, die Pbasenverschiebung des ersten Partialtons $36^{\circ}$, die des zweiten $2.36=72^{\circ}$, die des dritten $3.36=108^{\circ} \mathrm{u} . \mathrm{s} . \mathrm{w}$.

1) Dass ich den unteren, schon von $\mathrm{Hel} \mathrm{mholtz}$ behaupteten For. manten in meinen $\alpha$-Versuchen nicht gefunden habe, beruhte auf einem Mangel derselben, den ich selbst bereits hervorgehoben habe (Bd. 53. S. 18, 34 etc.), was $\mathbf{P}$ i p p in $\mathrm{g}$, nach der Art seiner Aeusserung zu schliessen, übersehen zu haben scheint. 


\begin{tabular}{|c|c|c|}
\hline . & $\begin{array}{l}\text { Zahl der Fälle } \\
\text { mit Amplituden- } \\
\text { maximum beim } \\
\text { Grundton }\end{array}$ & $\begin{array}{c}\text { Zahl der Fälle } \\
\text { mit Amplituden- } \\
\text { maximum bei } \\
\text { einem höheren } \\
\text { Partialton }\end{array}$ \\
\hline $\begin{array}{l}\text { in Pipping's ersten Analysen } \\
\text { in Pipping's neuen Ana- } \\
\text { lysen }{ }^{1} \text { ). }\end{array}$ & $\begin{array}{l}14 \\
29\end{array}$ & $\begin{array}{r}9 \\
39\end{array}$ \\
\hline $\begin{array}{l}\text { meinen ersten Analysen }{ }^{2} \text { ) } \\
\text { ( } \leftrightarrow \text {-Vers., Bd. 47) } \\
\text { in einer zweiten Reihe } \\
\text { (Bd. 53) } \\
\text { in miner dritten Reihe } \\
\text { (Bd. 58) . . . . . . }\end{array}$ & $\begin{array}{l}7 \\
2\end{array}$ & $\begin{array}{l}12 \\
36 \\
36\end{array}$ \\
\hline
\end{tabular}

Pipping hat sich also meinen Befunden beziglich des Grundtones erheblich genähert, und betrachtet sogar den von mir zuerst, im Gegensatz zu den älteren Arbeiten, nachdrücklich hervorgehobenen merkwïrdigen Umstand, dass die Vocalklänge auffallend schwache Grundtöne haben, jetzt wie eine bekannte Thatsache (S. 535). Trotzdem ist aber der Unterschied in unseren Resultaten in dieser Hinsicht noch recht beträchtlich. Ein Grund hiervon mag in der im Allgemeinen böheren Láge von Pipping $g^{1} s$ Vocalnoten liegen. Aber zum Theil liegt der Grund vielleicht auch in den verwendeten Membranen. Pipping erhebt (S. 557) „Protest“ dagegen, dass ich schon früher vermuthet habe, die Abweichnngen möchten nur zu einem kleinen Theile auf Verschiedenheit der Aussprache beruhen. Ich muss aber meine Vermuthung aufrecht erhalten; grade für d ies e n einigermassen principiellen Punkt kan n die Aussprache gar nicht in Betracht kommen. Mein Verfahren muss ich nach wie vor als das- zuverlässigere erklären, weil das Abhören der Eingrabungen am Edison'schen Phonographen jederzeit über die Trene derselben Aufschluss giebt, eine Controle, welche beim Sprachzeichner nun einmal unmöglich ist. Wenn letzterer etwa bei gewissen Vocalen "die Grundschwingung besonders begünstigte, so wäre die Verschiedenheit der Ergebnisse erklärlich. Allerdings sucht Pipping meine Methode zu verdächtigen. Nachdem er nämlich die längst bekannte Thatsache constatirt hat, dass der

1) Es sind in dieser Uebersicht nur die gesungenen Vocale berücksichtigt.

2) Nur Vocal $A$ betreffend. 
Phonograph nur dann treu die Vocale wiedergiebt, wenn man mittels der Obrschläuche hört, schliesst er nicht etwa, wie man erwarten sollte, dass schlechte Wiedergabe an sich nocb Nichts gegen die Treue der Eindrücke beweist, sondern umgekehrt (S. $555 \mathrm{f}$ ): "Trotz der guten Wiedergabe der Vocale müssen wir uns deswegen sehr hüten, die Richtigkeit der" Eindrücke auf dem Wachscylinder als direct controlirt zu betrachten." Pip ping scheint es also für denkbar zu halten, dass falsche Eingrabungen durch Behorchen mit einem Schlauch zu richtigen Vocaleindrücken führen! Unéndlich näber liegt doch die Erklärung, dass die Vocaleingrabungen richtig sind, beim Behorchen ohne Schlauch aber nicht so genau mit allen ihren Specialitäten auf das Ohr wirken, als mit Schlauch ${ }^{1}$ ).

Pipping's ;Verstärkungsgebiete" erstrecken sich zum Theil iiber Bereiche von einer vollen Octave und mehr. Da diese enorme Ausbreitung ausscbliesslich die tiefsten Gebiete betrifft, deren Mitteipunkte in die 1-gestrichene Octave fallen, so könnte das Hervorragen der Grundtöne an Pipping's Schlïssen wesentlichen Antheil haben.

Zur genaueren Ermittelung der Centren seiner Verstärkungsgebiete hat sich Pipping jetzt meiner Idee einer Schwerpunktsberechnung angeschlossen, aber dieselbe zu einer logarithmischen umgestaltet. Sind $p_{1}, p_{2}, p_{3}$ die Amplituden für die Ordnungszahlen $n_{1}, n_{2}, n_{3}$ ete., so rechne ich als resultirende (unharmonische) Ordnungszahl:

$$
n=\frac{p_{1} n_{1}+p_{2} n_{2}+p_{3} n_{3}+\ldots}{p_{1}+p_{2}+p_{3}+\ldots}
$$

Pipping dagegen rechnet $\left.{ }^{2}\right)$ :

$$
\log n=\frac{p_{1} \log n_{1}+p_{2}}{p_{1}}+\frac{\log n_{2}+p_{3}}{+p_{2}} \frac{\log n_{3}+\ldots}{+p_{3}}+\ldots
$$

woraus folgen würde:

$$
n=\frac{\left(p_{1}+p_{2}+p_{3}+\ldots\right)}{-\sqrt{n_{1}^{p_{1}} \eta_{2} \eta_{2}^{p_{2}} n_{3}{ }^{p_{3}}} \ldots}
$$

1) Die frühere, vom Sprachzeichner hergenommene Ansicht, dass der S p rech schlauch ungünstig wirke, ist hoffentlich, nachdem $\mathrm{P}$ i p ping den Phonographen näher kennen gelernt hat, von ihm aufgegeben.

2) Statt der Ordnungszahlen nimmt Pipping die entsprechenden Schwingungszahlen, was aber im Resultat auf das Gleiche herauskommt. 
Seine Begründung läuft darauf hinaus, dass der intervallmässig mittlere Ton zwischen zwei Tönen nicht beim arithmetischen, sondern beim geometrischen Mittel der Schwingungszahlen liegt. Das ist ganz richtig; nur hat Pip ping versäumt, nach zuw ei se $n$, dass es bier auf Intervallverhältnisse im musikalischen Sinn, und nicht auf einfache lineare Abstände der Schwingungszablen ankommt.

Will man der Sache physikalisch auf den Grund gehen, so wäre das nächstliegende, einen einigermassen analogen übersehbaren Fall zu untersuchen, z. B. in welchem Amplitudenverhältniss ein irgendwie gelegener Ton eine Reihe von Resonatoren, deren Schwingungszahlev sich wie die ganzen Zahlen verhalten, anspricht. Diese Aufgabe ist theoretisch vollkommen lösbar, und ich gebe bier kurz die Resultate der von mir ansgeführten Berechnung.

Für einen Resonator von der Eigenschwingungszahl $k$ für $2 \pi$ sec. und dem Dämpfungsverhältniss $\varepsilon$ (die Masse $=1$ gesetzt), auf welchen ein Ton von der Amplitude $\lambda$ und der Schwingungszahl $m$ (fiir $2 \pi$ sec.) einwirkt, lautet die Differentialgleichung:

$$
\frac{\partial^{2} x}{\partial t^{2}}+2 \varepsilon \frac{\partial x}{\partial t}+k^{2} x=\lambda \sin m t .
$$

Das Integral dieser Gleichung ist, wenn die mit der Zeit schnell verschwindenden Glieder weggelassen werden,' auf eine Sinusschwingung reducirt:

$$
x=\frac{\lambda}{\sqrt{\left(k^{2}-m^{2}\right)^{2}+4 m^{2} \varepsilon^{2}}} \sin \left(m t-\operatorname{arctg} \frac{2 m \varepsilon}{k^{2}-m^{2}}\right) .
$$

Der vor dem sin-Zeichen stehende Factor ist die Amplitude, mit welcher der Resonator antwortet, und wenn eine Reihe von Resonatoren von den Schwingungszahlen $k, 2 k, 3 k, 4 k$ etc. vorliegt, so wirkt der Ton $m$ in dem Verhältniss

$$
\frac{1}{\sqrt{\left(k^{2}-m^{2}\right)^{2}+4 m^{2} \varepsilon_{1}^{2}}}, \frac{1}{\sqrt{\left(4 k^{2}-m^{2}\right)^{2}+4 m^{2} \varepsilon_{2}^{2}}} \text {, etc. }
$$

auf sie ein, worin $\varepsilon_{1}, \varepsilon_{2}$ etc. die Dänpfungsverhältnisse sind.

Wenn nun Pipping's Begründung seines Verfahrens eine tiefere Bedeutung hätte, so müssten zwei Resonatoren, z. B. von der. Schwingungszahl $2 k$ und 37 , annähernd gleich angesprochen werden durch einen Ton, der dem g e o metrischen Mittel von $2 \%$ und $3 k$ entspricht, d. b. von $m=\sqrt{6} . k$. Me ine Schwerpunktsberechnung dagegen wiurde voraussetzen, dass diese beiden Resonatoren annähernd gleich angesprochen werden dureh den Ton vom arith- 
metischen Mittel, d.h. $m=2,5 k$. Nehmen wir die $\varepsilon$-Werthe so klein an, dass ibre Quadrate gegen die übrigen Glieder vernachlässigt werden dürfen, so spricht der nach $\mathrm{Pi}$ p ping berechnete Ton die beiden Resonatoren im Verhältniss von $3: 2$, der pach mir berechnete im Verhältniss von $11: 9$, also weit gleichmässiger, an. Man sieht also, wie wenig fundirt Pipping's Aperçu ist ${ }^{1}$ ).

Aber Pipping hätte eine noch viel näher liegenden Probe machen können, wenn er nachgesehen hätte, wie sich seine Rechnungsweise an völlig übersebbaren Beispielen analysirter Curven bewährt. In einem Anhange zu Theil IV meiner Arbeit (dies Archiv Bd. 53. S. 46-51) habe ich zar Prüfung meiner Schwerpunktsberechnung, welche ich ausdrücklicb stets (u. A. S. 51) als eine blosse Annäherung betrachtet habe, eine Anzahl Fälle von Perioden mit unharmonischen Bestandtheilen rechnerisch analysirt, in 3 Tabellen die Amplituden der Theilschwingungen dargestellt, und (S. 50) die unharmonischen Bestandtheile mit den Schwerpunktsermittelungen verglichen. Hier bätte $\mathrm{Pipping} \mathrm{ohne}$ jede Muilhe exactes Material zur Prüfung seines Aperçu's gefunden. Ich habe es mich die Mühe nicht verdriessen lassen, Pipping's Berechnung auf diese Beispiele anzuwenden. Es kommt heraus:

\begin{tabular}{|c|c|c|c|c|c|}
\hline $\begin{array}{c}\text { Tabelle } \\
\text { Nr. }\end{array}$ & $m=$ & $\begin{array}{c}\text { Berücksichtigte } \\
\text { Amplituden }\end{array}$ & $\begin{array}{l}\text { Wahrer } \\
\text { Werth }\end{array}$ & $\begin{array}{c}\text { Werth nach } \\
\text { meiner } \\
\text { Schwerp.-Ber. }\end{array}$ & $\begin{array}{l}\text { Werth nach } \\
\text { Pipping's } \\
\text { Schwerp.-Ber. }\end{array}$ \\
\hline 1 & $\begin{array}{l}2 \\
3 \\
4\end{array}$ & $\begin{array}{l}4,5,6 \\
4,5,6 \\
4,5,6\end{array}$ & $\begin{array}{l}5,0 \\
5,0 \\
5,0\end{array}$ & $\begin{array}{l}4,94 \\
4,95 \\
4,97\end{array}$ & $\begin{array}{l}4,88 \\
4,90 \\
4,93\end{array}$ \\
\hline 2 & $\begin{array}{l}2 \\
3 \\
4 \\
4,8\end{array}$ & $\begin{array}{l}4,5,6 \\
4,5 \\
4,5 \\
4,5,6\end{array}$ & $\begin{array}{l}4,8 \\
4,8 \\
4,8 \\
4,8\end{array}$ & $\begin{array}{l}4,87 \\
4,58 \\
4,68 \\
4,91\end{array}$ & $\begin{array}{l}4,71 \\
4,55 \\
4,65 \\
4,88 \mathrm{a}\end{array}$ \\
\hline 3 & $\begin{array}{l}2 \\
3 \\
4 \\
4,5\end{array}$ & $\begin{array}{l}3,4,5 \\
4,5 \\
4,5 \\
4,5\end{array}$ & $\begin{array}{l}4,5 \\
4,5 \\
4,5 \\
4,5\end{array}$ & $\begin{array}{l}4,09 \\
4,47 \\
4,47 \\
4,53\end{array}$ & $\begin{array}{l}4,09 \mathrm{~b} \\
4,52 \mathrm{c} \\
4,44 \\
4,50 \mathrm{~d}\end{array}$ \\
\hline
\end{tabular}

1) Ich bemerke ausdrücklich, dass auch für andere Annahmen bezüglich $\varepsilon$ die lineare Schwerpunktsberechnung meist richtigere Werthe liefert als Pipping's logarithmische. Ich habe in vielen Beispielen die Amplitudenverhältnisse für ein gegebenes $m$ ausgerechnet, und aus ihnen $m$ nach beiden Verfahren zu bestimmen gesucht. 
Wie man sieht, führt Pipping's sehr umständliche Rechnung in 7 von.11 Fällen auf unrichtigere Ergebnisse als die meinige, in 1 Falle (b) auf gleiches Ergebniss, und nur in 3 Fällen $(\mathbf{a}, \mathbf{c}, \mathbf{d})$ auf ein um 0,01 bis 0,03 richtigeres. Es existirt also weder ein theoretischer, noch ein practischer Grund, meiner einfachen Rechnung eine complieirtere und in der grossen Mehrzabl der Fälle geringere Annäherung ergebende Rechnung vorzuziehen; Pipping war und ist auch hier mit seinem Angriff im Unrecht, und hat sich überflüssige Mühe mit seiner Rechnung gemacht.

\section{Curven und Analysen kurzer Vocale} (h i e rzu Taf. VI).

Zum ersten Male gebe ich auch eine Sammlung von Curven kurzer Vocale (wie in Ann, Enn u. s. w.). Bei der kurzen Dauer derselben können im Allgemeinen auch nur kurze Periodenreihen gewonnen werden. In der Regel benutzte ich zur Aufzeichnung nur Viertel-Umdrehnngen des Cylinders (mittels des S. 171 erwähnten Vorsteckers), gewann also Curven von $12 \frac{1}{2} \mathrm{~cm}$ Länge. Selbst in diesen kurzen Strecken sieht man die Vocalperioden meist in Veränderung begriffen. Es liegt anscheinend in der Natur des kurzen Vocals, seinen Charactergegen das Ende, wo er fast stets in einen Consonanten, oder (zur Diphthongenbildung) in einen anderen Vocal übergebt, zu ändern; der Mund macht offenbar schon wähirend des Vocals Vorbereitungen zum Uebergang in die Stellung fiir den folgenden Laut, was nicht ohne Einfluss auf den Vocalcharacter bleiben kann. Die kurzen Vocale sind deshalb eine weniger constante Erscheinung als die langen, und auch in den Analysen mehrerer Perioden desselben Vocals zeigt sich geringere Uebereinstimmung der Resultate. Von anderen Autoren mit Ausnahme Boeke's ${ }^{1}$ ) sind bisher meines Wissens die kurzen Vocale von den entsprechenden langen nicht unterschieden worden. In Pipping's analysirten Vocalen scheinen sich, nach den angeführten Wortbeispielen (a. a. 0. S. 558) zu schliessen, sowohl lange als kurze zu befinden.

Dass manche kurzen Vocale, obwobl sie mit demselben Zeichen geschrieben werden, ganz andere Laute sind als die ent-

1) Dies Archiv Bd, 50. S. $307 \mathrm{f}$. 
sprechenden langen, habe ich schon in friberen Mittheilungen kurz erwälnt. Namentlich ist das kurze $E$ in Helm offenbar dem $A e$ ungleich näher stehend als dem (langen) $E$; dies bestätigt aucb der Anblick der Curven, und die-Unmöglichkeit, einen Untersebied der Worte Hälm and Heln zu hören, wenn man nicht absichtlich einen solchen hervorzubringen sucht. Ebenso ist das kurze $O$ in Wort dem Ao ungleich verwandt als dem (langen) 0 . Der Unterschied des kurzen $I$ (in $B i l d$ ) vom langen $I$ ist mir noch früher an dem Aussehen der Curven aufgefallen als durch das Gehör, obwohl er auch so deutlich ist. Und auch bei $U, O e, U e$ ist der kurze Lant wesentlich verschieden vom langen; das kurze $O e$ (in können) entspricht einer Art von langem $O e$, welche im Deutschen ungebräuchlich, dagegen im Französischen sehr gewöhnlich ist. Unter allen Vocalen scheint $A$ am wenigsten durch die Kürze verandert zu werden; doch nähert es sich bej den meisten Personen etwas dem Ao.

Auf Tafel VI gebe ich Curven aller hier genannten kurzen Vocale auf die Noten $c, e, g, c^{1}$, wiederum in $8 \mathrm{~cm}$ langen $\mathrm{Ab}$ schnitten. $E$ und $A e$ sind nicht nnterschieden, ebensowenig $A o$ und $O$.

Herr Hirschfeld hat eine Reihe von Analysen der kurzen Vocale ausgeführt, deren Resultate ich im Folgenden mittheile. Wie immer bedeuten die Zahlen die Amplituden der Partialschwingungen in Procenten dér maximalen Curvenhöhe.

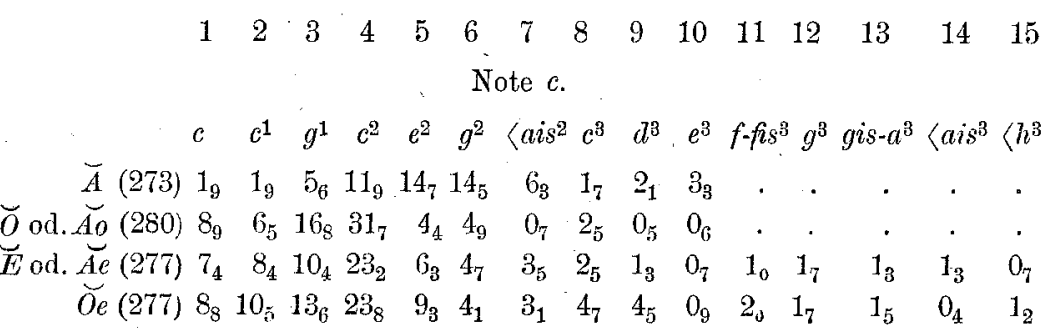

Note $e$.

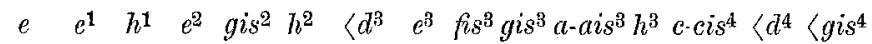

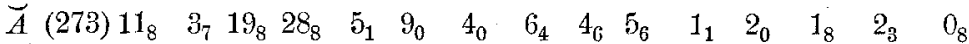

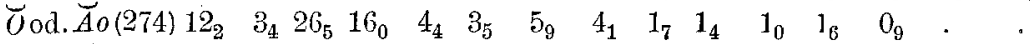

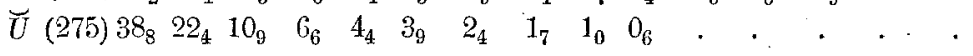

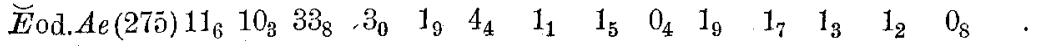

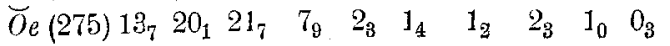

E. Pfüger, Archir f, Physiologie. Bd. 61 . 
Note $g$.

$\begin{array}{llllllllllllll}g & g^{1} & d^{2} & g^{2} & h^{2} & d^{3} & \left\langle f^{3}\right. & g^{3} & a^{3} & h^{3} & c-c i s^{4} & d^{4} & d i s-e^{4} & \left\langle f^{4}\right.\end{array}$ $\begin{array}{lllllllllllllll}\breve{A}(273) & 19_{2} & 6_{5} & 30_{7} & 10_{3} & 13_{4} & 1_{6} & 4_{5} & 6_{4} & 0_{9} & 2_{5} & 2_{1} & 2_{3} & 0_{9} & \text {. }\end{array}$ $\begin{array}{lllllllllllllll}\breve{O} \text { od. } \breve{A o}(274) & 14_{4} & 14_{8} & 31_{5} & 6_{2} & 5_{2} & 8_{9} & 3_{1} & 2_{7} & 3_{6} & 1_{4} & 2_{5} & 1_{7} & 0_{5} & 0_{3}\end{array}$

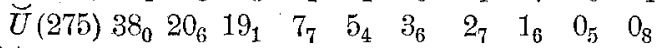

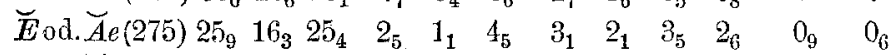

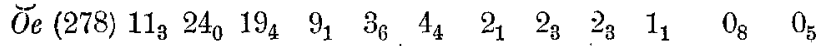

Note $c^{1}$.

$\begin{array}{rrrrrrrrrr}c^{1} & c^{2} & g^{2} & c^{3} & e^{3} & g^{3} & \left\langle\text { nis }{ }^{3}\right. & c^{4} & d^{4} & e^{4} \\ \widetilde{A}(273) 10_{0} & 43_{3} & 9_{4} & 10_{0} & 6_{9} & 2_{8} & 1_{3} & 1_{2} & 0_{5} & 0_{4} \\ \breve{U}(275) 15_{3} & 31_{2} & 15_{3} & 2_{8} & 3_{2} & 2_{1} & 0_{5} & 0_{4} & 0_{3} & 0_{3} \\ \widetilde{A e}(263) 11_{0} & 41_{0} & 8_{5} & 5_{9} & 2_{0} & 0_{4} & 0_{7} & 2_{3} & 0_{9} & 0_{6}\end{array}$

Aus diesen Analysen folgt zunächst, dass $\breve{U}$ von den iibrigen Vocalen und auch von $\bar{U}$ dadurch abweicht, dass die Partialamplituden von der tiefsten beständig und sebr gleichmässig abnehmen. Nur auf Note $c^{1}$ lässt sich ein bei $c^{2}$ liegender Formant herauslesen, von welchem auch auf Note $g$ eine Andeutung sich findet, da die dritte Amplitude $\left(d^{2}\right)$ von der zweiten kaum verschieden ist, während sonst die Amplituden gleichmässig abnehmen. $\breve{U}$ scheint also ein gewissen musikalischen Klängen sich annähernder Klang zu sein, in welchem ein bei $c^{2}$ liegender Formant wenig hervorragt.

$\widetilde{A}$ hat einen dentlichen Formanten in der zweiten Octave, wie $\bar{A}$, aber entschieden etwas tiefer. Die Schwerpunktsberechnung ${ }^{1}$ ) ergiebt seine Lage

$$
\begin{array}{clll}
\text { fiir } & \text { Note } & c: & \rangle e^{2} \\
" & \quad, & e: & e s^{2} \\
" & , & g: & \left\langle d i s^{2}\right. \\
" & " & c^{1} & c^{2}
\end{array}
$$

$\breve{O}(\breve{A} o)$ hat ebenfalls einen deutlichen Formanten, welcher tiefer liegt, als diejenigen von $\bar{A} 0$ and $\bar{O}$, nämlich nach der Schwerpunktsberechnung

$$
\begin{aligned}
& \text { für Note } c: b^{\mathbf{1}}-h^{\mathbf{1}} \\
& \text { " }, \quad e:>c i s^{2} \\
& \text { " , } \quad g:>c^{2}
\end{aligned}
$$

1) Ueber die Regel für die dazu auszuwählenden Amplituden s. dies Archiv Bd. 53. S. 50. 
$\breve{E}(\breve{A} e)$ zeigt ganz äbnlich aussehende Curven wie $\bar{A} e$; die Analysen zeigen die Uebereinstimmung in geringerem Grade. Für Ae lagen die Formanten im Anfang der 2, und in der Mitte der 3. Octave. Für $\breve{E}$ ergeben sich solche

$$
\begin{gathered}
\text { fuir Note } c: \quad c^{2} \text { und } g^{3}-a^{3} \text { (schwach) } \\
" \quad " \quad e: \quad h^{1}, h^{2} \text { und } f s^{3} \\
" \quad " \quad g: d^{2}, d i s^{3} \text { und } a^{3} \\
" \quad " \quad c^{1}: c^{2}, c^{4} .
\end{gathered}
$$

Formanten von $\breve{O} e$ ergeben sich aus den Analysen

$$
\begin{aligned}
& \text { für Note } c: c^{2}, c i s^{3}, g^{3} \\
& \text { " }, e: g^{1} s^{1}, e^{3} \\
& " \quad, \quad g: h^{1}, d^{3} \text {. }
\end{aligned}
$$

Der höhere Formant von Ŏ ${ }_{e}$ lässt sich besser durch Proportionalmessung ermitteln, und liegt nach zahlreichen solchen in der Gegend von $e^{3}$ bis $f i s^{3}$.

Für $\breve{U}$ e ergeben die Proportionalmessungen einen Formanten zwischen $f s^{3}$ and $g i s^{3}$.

\begin{tabular}{|c|c|c|}
\hline & langer Vocal & kurzer. Vocal \\
\hline $\begin{array}{l}A \\
E \\
I \\
O \\
A o \\
U \\
A e \\
O e \\
U e\end{array}$ & $\begin{array}{l}\text { Mitte der 2. Octave }\left(f^{2}-a^{2}\right) \\
\text { Anfang d. 2. u. Ende d. } 3 . \text { Octave } \\
\text { Mitte d. 4. Octave } \\
\text { Anfang "2. " } \\
\text { Anfang der 1. und der 2. Octave } \\
\text { Mitte der" 3. Oct. (etw. tiefer als Ae) } \\
\text { Ende der 3. Octave }\end{array}$ & $\begin{array}{l}\text { Anfang d. 2. } \begin{array}{l}c^{2}-e s^{2} \\
a i s^{3} \\
b^{1}-c i s^{2} \\
c^{2} ?\end{array} \\
\text { Anfang d. 2. u. Mitte d. 3. Octave } \\
e^{3}-f s^{3} \\
\text { fis } s^{3}-g i s^{3}\end{array}$ \\
\hline
\end{tabular}

Endlich für $\widetilde{I}$ ergiebt sich auf demselben Wege aus sehr zahlreichen Proportionalmessungen ${ }^{1}$ ) gut übereinstimmend ein Formant bei ais ${ }^{3}$.

Schliesslich mögen die von mir für die langen und kurzen Vocale gefundenen Formantlagen übersichtlich zusammengestellt werden.

Im Allgemeinen liegen also die Formantender kurzen Vocale, besonders die böheren, tiefer

1) Vom kurzen $I$ besitze ich eine ungemein grosse Zahl von Curven, weil dieser Vocal in meinen Consonantuntersuchungen überwiegend als Silbenbilduer verwendet wird (vgl. dies Archiv Bd. 58. S. 260). 
als diejenigender entsprechenden langen Vocale. Dieses Ergebniss bestätigt sich rollkommen auch beim Flüstern der langen und kurzen Vocale.

5. Zur Theorie der Vocalbildung.

Nachdem durch meinen Geschwindigkeitsversuch am Phonographen ${ }^{1}$ ) und kurz darauf durch meine und Pipping's von einander unabhängige Curvenanalysen die lange Zeit offene Frage, ob die Vocalformanten feste oder bewegliche Lagen haben, im Sinne der Helmholtz'schen Ansicht von 1863 entschieden war, trat die zweite Frage in den Vordergrund, wie die festen Formanten sich mit den variirenden Stimmklängen abfinden.

Helmholtz hatte diese Frage dahin beantwortet, dass der Mundresonator, dessen Eigenton der Formant ist, die dem letzteren zunächst liegenden Partialtöne des Stimmklanges verstärke, und diese Ansicht hat neverdings in P i p p ing einen eifrigen Vertreter gefunden. Ursprïnglich ${ }^{2}$ ) hatte er Helmholtz's Annahme, dass die Verstärkungsgebiete sich iiber eine Octave und mehr (2 Quinten) erstrecken können, bestritten, jetzt ${ }^{3}$ ) geht er sogar iiber diese weite Ausdehnung hinans.

Abgesehen davon, dass bei dieser Sachlage der Vocal mit besonderer Vollkommenheit auf diejenigen Noten sich singen lassen miisste, mit deren Obertönen der Formant übereinstimmt, habe jch gegen die Verstärkungstheorie bereits eingewendet ${ }^{4}$ ), dass, besonders bei Männerstimmen, die Formanten vieler Vocale auf viel zu bohe Partialtöne des Stimmklanges fallen, als dass man annehmen könnte, dass dieselben überhaupt vertreten sind, also verstärkt werden können. So ist der so sehr ausgeprägte Formant des langen Vocals $I\left(\mathbf{f}^{4}\right)$

$$
\text { bei Note } G \text { der 28.-29. Partialton, }
$$

$$
\begin{array}{llllrl}
" & , & c & , & 21-22 . &
\end{array}
$$

Dieser Einwand scheint mir schon genïgend, um die Theorie unmöglich zu machen.

1) Dies Archiv Bd. 47. S. 42.

2) Zeitschr. f. Biologie Bd. 27. s. 75.

3) Ebendaselbst Bd. 31. S. 573, 583.

4) Dies Archiv Bd. 58. S. 274. 
Ferner wende ich ein, dass die auf diese Theorie gegründeten Vocalsynthesen misslingen, während die auf die meinige gegründeten den frappantesten Erfolg haben (s. unten). Wer je versucht hat, durch Aufsetzen von Resonatoren von der Tonhöhe $f^{2}$ bis gis ${ }^{2}$ auf eine Zungenpfeife den Vocal $A$ zu produciren, wird zugeben müssen, dass dies nicht gelingt, geschweige denn dass sich auf diesem Wege $E$ oder $I$ hervorbringen liessen. Ferner miisste nach Pipping's Theorie der Vocal $A$ beim Helmholtz'schen Klavierversuch auch dann als solcher nachhallen, wenn man statt die Dämpfer mittels des Pedals sämmtlich abzuheben; nur die Saiten $g^{2}$ oder gis ${ }^{2}$ oder selbst alle Saiten des "Verstärkungsgebietes" durch Niederhalten ihrer Tasten von den Dämpfern befreit. Nie hört man so den Vocal. Ebensowenig hört man ibn, wenn man die Tasten sämmtlicher Obertöne der gesungenen Note, soweit sie vertreten sind, niedergedriickt hält. Alle diese leicht zu bestätigenden Thatsachen sind unüberwindliche Schwierigkeiten für die Verstärkungstheorie. Zur Reproduction der Vocale müssen schlechterdings alle Saiten sammt ihren Obertönen frei sein, damit alle Töne, welche aus der Analyse der Periode mit anaperiodischer Theilschwingung hervorgehen, ihre Vertretung finden können (s. oben S. 178). Wie sehr es übrigens bei diesem Versuch nicht blos auf die Grundtöne, sondern auch anf die Ohertöne der Saiten ankommt, geht aus folgenden von mir beobachteten Thatsachen hervor. Erstens gelingt der Versuch auch sehr gut an einem total absichtlich verstimmten Klavier. Zweitens gelingt er ziemlich gut auch dann, wenn man das Pedal erst im Augenblick des Aufhörens des gesungenen Vocals niedertritt; letzteres kann nur darauf beruhen, dass auch die gedämpften Saiten noch Partialscbwingungen ausführen können, welche sich nachher durch Abheben des Dämpfers etwas verstärken.

Weiter habe ich bereits früher gegen die Verstärkungstheorie (und für meine Auffassung) den Umstand angefiuhrt, dass bei der telephonischen und microphonischen Uebertragung die Vocale sich ganz anders verhalten als sonstige Klänge $\left.{ }^{1}\right)$. Unter solchen Umständen, dass die Amplitudenverhältnisse durch Multiplication odel Division mit den Ordnungszahlen oder sogar mit den höheren Potenzen derselben, sich total ändern, werden, wie ich gezeigt habe, die Klänge gänzlich deformirt, die Vocale behalten aber ihren Character,

1) Dies Archiv Bd 48. S. 543. 
und zwar weil trotz jener Veränderungen des Amplitudenverhältnisses die Grundform der Vocalcurve erhalten bleibt. Ich schloss hieraus, dass es für den Vocalcharacter eben auf diese characteristische Eigenschaft, nämlieh die Wiederholung einer bestimmten Oscillationsgruppe in jeder Periode, und nicht auf das Amplitudenverhältniss der Partialtöne ankommt wie bei musikalischen Klängen, deren Character sich bei diesen Versuchen in unbeschreiblichem Grade ändert.

Mit diesen Thatsachen sucht sich Pipping einfach dadurch abzufinden, dass er meint, die nach meiner Rechnung sich ergebenden Aenderungen der Amplitudenverbältnisse seien gar nicht so besonders gross, um den Klang wesentlich zu ändern; insbesondere bleibe das "Verstärkungsgebiet" immer noch prominent genug. Er glaubt dies an einem Beispiel, das er ausgerechnet hat, nachweisen zu können. Wer die Zahlen dieses Beispiels unbefangen betrachtet, wird sich vermuthlich über $P$ ipping's Urtheil wundern. Noch dentlicher treten die Aenderungen bervor, wenn man in den verglichenen Zahlen nicht die Summe, sondern die hervorragendste Formant-Amplitude $=100$ setzt. Ich habe das erste beste Beispiel aus meinen Analysen, nämlich das zweite $A$ auf Note $c$ in der Zusammenstellung in diesem Arehiv Bd. 53. S. 22 (das erste $A$ habe ich, weil es auf 15 Amplituden analysirt war, nicht gewählt), in dieser Weise ausgerechnet, gewiss nicht grade das gunstigste für das, was zu zeigen ist, und stelle die Zahlen hier zusammen:

\begin{tabular}{r|r|r|r|r}
\hline \hline & \multicolumn{1}{c}{$\begin{array}{c}\text { A. } \\
\text { B }\end{array}$} & $\begin{array}{r}\text { B. } \\
n\end{array}$ & $\begin{array}{c}\text { C. } \\
n^{2} p\end{array}$ & \multicolumn{1}{c}{$\begin{array}{c}\text { D. } \\
p / n\end{array}$} \\
\hline 1 & 22,0 & 4,4 & 0,9 & 110,1 \\
2 & 29,5 & 11,8 & 4,7 & 73,7 \\
3 & 7,5 & 4,5 & 2,7 & 12,5 \\
4 & 27,2 & 21,8 & 17,4 & 34,0 \\
5 & 100,0 & 100,0 & 100,0 & 100,0 \\
6 & 53,0 & 63,6 & 76,3 & 44,2 \\
7 & 20,5 & 28,7 & 40,2 & 14,7 \\
8 & 28,0 & 44,8 & 71,6 & 17,5 \\
9 & 17,2 & 30,9 & 55,6 & 9,5 \\
10 & 6,7 & 13,4 & 26,9 & 3,4 \\
& & & &
\end{tabular}

Die Verticalcolumne $A$ entspricht den Amplitudenverhältnissen des urspriinglichen Vocals, $B$ einer einfachen telephonischen Ueber- 
tragung bei relativ grossem Widerstand, $C$ einer telephonischen Uebertragung mit eingeschalteter Induction, ebenfalls bei grossem Widerstande, $D$ der gewöhnlichen Uebertragung mit Microphon, Inductionsspule und Telephon bei relativ kleinem Widerstande. Bei $C$ und $D$ bleibt der Vocal gleich gut erkennbar. Wer die Zahlen unter $C$ und $D$ vergleicht, wird mit mir schliessen, dass es auf die Amplitudenverhältnisse nicht ankommt, sondern nur auf das Vorhandensein einer bestimmten Schwingung, nämlich derjenigen des Formanten. Pipping's entgegengesetzter Schluss ist mir unverständlich, zumal er viel auf die Intensitäten giebt, und in diesen die Entstellung durch Microphon und Telephon noch viel grösser wird. Es wäre zu wïnschen, dass Pipping meine Versuche uiber Telephon etc. wiederholte; er wïrde dann sich iberzeugen, wie ungeheuer verschieden der Einfluss auf musikalischen Klang und auf Vocale ist.

Von ähnlicher Bedeutung wie die Microphon- und Telephonversuche sind für meine Auffassung der Vocalnatur gewisse am Phonographen von mir häufig gemachte Beobachtungen. Arbeitet nämlich der Recorder beim Singen etwas zu seicht auf dem Wachscylinder, so graben sich nur die hervorragendsten Theile der Schwingung ein, und bei den Zurückweichungen der Glasmembran bleibt das Wachs unverletzt. Die Curven soleher Eingrabungen, von denen ich sehr viele besitze, zeigen dann statt der unteren Gipfel gradlinige horizontal abschneidende Linien. Trotzdem hört man beim Reproduciren solcher Eingrabungen die Vocale $A, A o, O$ mit unverminderter Deutlichkeit; man sieht also, dass es bei den Vocalen eben nur auf die Abstände der Gipfel ankommt. Natürlich geben solche Curven bei der Analyse eine wesentlich andere Amplitudenvertheilung, obne aber die Formanthervorragung zu verwischen. Um hiervon ein deutliches Bild zll erhalten, habe ich von Herrn. Hirschfeld eine bereits von ihm gemessene und analysirte Curve dadurch in eine von der beschriebenen Art verwandeln lassen, dass die oberen Gipfel abgeschnitten wurden; einfach dadurch dass alle einen bestimmten Grenzwerth ïberschreitenden Ordinaten (die Nulllinie lag an den unteren prominentesten Gipfeln) auf diesen Grenzwerth vermindert wurden. Die so veränderten Ordinatenwerthe wurden nun der Analyse unterworfen, wobei sich das erwartete Resultat zeigte.

Man kann sich übrigens solche unvollständige Eingrabungen 
wie die bezeichneten auch künstlich herstellen, indem man einen Cylinder mit vollständigen Vocaleingrabungen soweit abdreht, dass nur noch die tiefsten Stellen der Eingrabung bestehen bleiben. Lässt man einen solchen Cylinder mittels des Reproducers sprechen, so hört man vièle Vocale, namentlich $A$, und anch die energischeren Consonanten, ungemein dentlich. Ich ziebe aus allen diesen Erfahrungen, welche sich mir bei meinen Arbeiten von selber anfgedrängt haben, denselben Schluss, wie aus meinen Telephon- und Microphonversuchen, dass nämlich für die Sprachlaute Stösse, welche in gewisser Zeitfolge eintreten, massgebender sind, als die Gesammtheit des Klanges, wie er sich in der Analyse nach harmonischen Partialtönen darstellt.

Meine eigene Auffassung von der Natur der Vocale erblickt in den Vocalen eine ganz specifische Art von Schall, deren Natur erst zu ergrïnden ist, und nie ergrïndet werden wird, wenn man mit aller Gewalt ihr die geläufigen Begriffe der Instrumentalmusik oder gar die anscheinenden Erfordernisse einer Hörbypothese aufzwängen will. Ist es nicht bemerkenswerth, dass wir die näcbsten Analoga der Vocale nicht in der Musik, sondern in der Welt der Geräusche finden, dass zahlreiche Geräusche, wie Knattern, Schmettern, Donnern, Klirren ihre Benennung von Anklängen an Vocale erhalten haben, und zwar in allen Sprachen? Welches musikalische Instrument klingt dagegen $A$-artig? Und doch sollte man dies wenigstens für gewisse Notenlagen bei irgend einem erwarten, wenn es blos auf Amplitudenverhältnisse der Partialtöne ankäme.

Ich sehe nun in den Curven anaperiodische Schwingungsgruppen von grosser Selbstständigkeit, welche von der Stimmnote unabbängig sind, und höchstens mit ihr ein klein wenig in die Höhe gehen. Diese Gruppen sind im Allgemeinen unbarmonisch zur Stimmperiode, und nur ausnahmsweise, gleichsam zufällig, harmonisch. Sobald ich künstlich einen ähnlichen Vorgang erzeuge, z. B. auf dem Wege der Interferenz, oder der Intermittenz, höre ich Vocale so frappant, wie mit keiner anderen mir bekannten Productionsmethode. Ich kann nur bedauern, dass Pipping nicht meinen hier so Vielen gezeigten Vocalversuch an der Doppelsirene wiederholt hat, obwohl ibm doch sicher, sei es in Kiel oder in 
Helsingfors, die Mittel dazu zu Gebote standen, und obwobl er früher nichtssagende Einwände dagegen erhoben hat.1). Selbst den weit unvollkommneren Zahnradversuch hat er nicht angestellt, obwohl er, um mich anzugreifen, mit Zahnrädern eifrig gearbeitet hat (s. unten). Wenn eine Betrachtung so spielend leicht zu einer früher unbekannten Art Vocale nachzuahmen gefübrt hat, so verdient sie mehr Beachtung, als ihr Pipping widmet.

Zu seiner Entschuldigung lässt sich anfiuhren, dass er einem Versuch ron Hensen zu grosse Bedeutung beigelegt hat, der sich gegen eine Idee von mir, wie die Vocale entstehen könnten, wendet. Pipping stellt diesen Versuch sogar unter seinen Gegengriinden in die erste Linie, ohne zu bedenken, dass es sich nur um eine von mir mit aller Reserve hingestellte Möglich k eit handelt. Seḷbst wenn diese Möglichkeit zurückzuweisen wäre, und wenn ich absolut nicht erklären könnte, wie die von mir behauptete Vocalerscheinung: zu Stande kommt, wäre doch diese Erscheinung damit nicht bestreitbar. Seit wann bezweifeln wir z. B. morphologische Erscheinungen deswegen, weil wir sie nicht zu erklären vermögen?

Ich hatte in meiner ersten Vocalarbeit gesagt ${ }^{2}$ ): „Das Wesentliche des Vocals wäre nach meinen Versuchen ein intermittirendes oder oscillirendes Anblasendes Mudtones durch die Stimme. Wenigstens genügt dies zur Characteristik des Vocals, und das Genügende, d. h. Notbwendigste festzustellen, muss unsere erste Aufgabe sein. . . . . Dass ein intermittirendes Anblasen iuberhaupt eintritt, scheint mir nicht unverständlich. Wir haben es vermöge der Schwingung der Stimmbänder mit einem intermittirenden Exspirationsstrom zu thun, welcher anblasend wirkt. Die Theorie der Resonatoren ist noch nicht genïgend entwickelt um übersehen zu können, unter welchen Umständen intermittirendes oder oscillirendes Anblasen ein continuirliches, und unter welchen es ein oscillirendes Tönen hervorbringt, und $\mathrm{n}$ a mentlich sind bekanntlich die Eigenschaften starrer und regelmäsig. geformter Resonatoren nicht obne Weiteres massgebend für die complicirte Form und dieweichen dicken. Wände des Mundresonators, der vielleicht viel stärker gedämpft ist" ${ }^{* 3}$ ).

1) Vgl. hierüber dies Archiv Bd. 48. S. 189.

2) Dies Archiv Bd. 47. S. 380, 381.

3) Wie ich aus der mir erst später zu Gesichte gekommenen 4. Ausgabe 
Gegen diese, wie man sieht mit aller Reserve ausgesprochene Hypothese rom Zustandekommen der Vocale, in weleher namentlich in den gesperten Worten (im Original nicht gesperrt) a uf die unbekannten Eigenschaften des Mundresonators Gewicht gelegt wird, hat $\mathrm{Hensen}$ einen Versuch mit einem gewöbnli chen starren Resonator producirt ${ }^{1}$ ), der fürPipping's neuen Angriff die wesentliche Stiitze bilden soll. Ich babe es bisher unterlassen mich zu dieser Hen s e n' schen Mittheilung zu äussern, weil der Herr Verfasser so freundlich war, einen Theil der darin enthaltenen Angriffe zuriuckzunehmen ${ }^{2}$ ), und ich es vorzog, das nicht Zurückgenommene auf sich beruhen zu lassen. Auch heute beschränke ich mich auf den in Rede stehenden Versuch.

Bekanntlich lässt sich ein Hohlkörper, z. B. eine Flasche, ein Kugelresonator, zum kräftigen Tönen bringen, wenn man einen Luftstrom aus einer passend gestellten Spalte über die Mündung streichen lässt. H e n se n bringt nun mit der oberen Oeffnung einer Zungenpfeife ein Spaltrohr in Verbindung und lässt die aus letzterem ausströmende Luft auf einen Resonator wirken; derselbe tönt nicht, wenn die Zunge schwingt, wohl dagegen, wenn die Luft durch die Zunge geht, obne dieselbe zum Schwingen zu bringen. Hensen schliesst hieraus, dass eine tönende Luftlamelle einen Resonator nicht anblasen kann.

Ich zweifle nicht an der Richtigkeit der Hen sen'schen Beobachtung, wohl aber an ihrer Allgemeinguiltigkeit, und an der Richtigkeit des gezogenen Schlusses. Mir ist bei zahlreichen Wiederholungen und Modificationen des Hensen'schen Versuches nur zuweilen ein ähnliches Resultat begegnet. Jedenfalls ist es sehr leicht, sich rom Gegentheil des Hensen'schen Satzes durch die einfachsten Versuche zu überzeugen.

Der Resonator mit Spaltrohr ist nämlich ein relativ unbequemes und unsicheres Mittel zur Bervorbringung eines Tones, wir haben aber in jeder Labialpfeife einen genau nach demselben Princip wirkenden ungleich bequemeren Apparat. Auch, hier wird am Labium Luft durch einen Spalt an der Mündung eines Hohlraums vor-

der "Tonempfindungen" (1877. S. 182) ersehen habe, schreibt schon $\mathrm{H}$ e l m holtz den Mundresonatoren eine ungewöhnlich starke Dämpfung zu.

1) Zeitschr. f. Biologie Bd. 28. S. 39.

2) Ebendaselbst S. 227. 
beigeblasen; nur ist der Hohlraum hier in sehr passender Weise fest mit dem Spalt verbunden, indem die bewegte Luftlamelle längs einer schiefen Ebene geleitet wird, und ferner ist. der Hohlraum meist parallelepipedisch oder cylindrisch statt kuglig. Bringt man nun eine Labialpfeife mit ihrem Mundstück luftdicht auf die Mündung einer Zungenpfeife, so wird sie mit Leichtigkeit jedesmal mit zum Tönen gebracht, wenn die Zunge tönt. Dabei ist es gleichgültig, ob die Labialpfeife offen oder gedeckt ist, und in welchem Verhältniss ihre Note zu derjenigen der Zunge steht, Sind beide Noten einander nahe genug, so entstehen die schönsten Sehwebungen beider Töne.

Hensen behauptet auch; dass man den Resonator durch ein Spaltrohr nicht zum Tönen bringen kann, wenn man letzteres mit dem. Munde anbläst, und gleichzeitig die Stimme wirken lässt, woll aber, wenn das Anblasen stimmlos erfolgt. Auch hier zeigt die. Labialpfeife das entgegengesetzte Verhalten; sie tönt, mit dem Munde angeblasen, stets, gleicbgültig ob mit oder ohne Stimme.

Hensen's Satz, dass eine schwingende Luftlamelle einen Hohlraum nicht anblasen kann, ist also unrichtig, wie er auch theoretisch unverständlich sein würde; und damit fällt sein und Pipping's Einwand gegen meine Idee von der Vocalbildung.

Freilich ist bei den Versuchen mit der Labialpfeife der Ton derselben völlig continuirlich, und giebt demgemäss auch dem Zungenklange keine Annäherung an den Vocalcharacter, wenn man die Labialpfeife in der. Note des Vocalformanten wählt. Wer hierin einen Einwand gegen meine Idee von der intermittirenden oder oscillirenden Anblasung der Mundhöble erblicken will, mag es thun. Aber es ist doch gewiss näherliegend, zu erwägen, dass weder die Klänge der Zunge mit derjenigen des menschlichen Kehlkopfs identisch sind, noch der starrwandige Hohlraum eines Resonators oder einer Pfeife verglichen werden kann mit dem dickund weichwandigen und unregelmässig geformten Munde, über dessen Dämpfungsgrad wir Nichts wissen (s. oben S. 193 f.) und dass vor Allem in den hier in Rede stehenden Versuchen eine Spalte zwischen Zunge und Resonanzraum angebracht ist, welche bei der Vocalbildung kein Analogon hat. Dass aber Spalten die vorhandenen Druckoscillationien zu amortisiren geeignet sind, darüber kann kein Zweifel sein.

Bis auf Weiteres ist also meine Idee, dass die Oscillationen 
des phonischen Exspirations- (oder Inspirations-) Stromes den Mundraum zu oscillirenden Tönen bringen, nicht widerlegt. Ich wiederhole, dass ich auf dieselbe keinen grossen Werth lege und dass am wenigsten meine Auffassung der Vocale mit derselben steht und fällt.

Für die Theorie, dass der Mundresonator dureh den Stimmklang continuirlich angeblasen wird, führt $\mathrm{Hel}$ inholtz in der 4. Ausgabe seiner "Tonempfindungen“ (1877, S. 184) folgendes an. Bei der Einwirkung eines Tones auf einen Resonator antwortet dieser nach der Theorie zuerst neben dem einwirkenden Tone mit seinem Eigentone, welcher aber wegen des Factors $e^{-s t}$, worin $\varepsilon$ die Dämpfungsconstante, schnell erlischt. Helmholtz giebt nun an, dass man beim scharfen Einsetzen eines Vocals wirklich anfangs den Mundton deutlich heraushört. Ich habe mir viele Mühe gegeben, diese Beobachtung zu bestätigen; es war mir aber nicht möglich.

\section{Zur Theorie des Hörens der Vocale.}

Die drei Fragen des objectiven Wesens der Schallbewegung bei den Vocalen, der Entstehung derselben, und endlich ihrer Wirkung anf das Ohr, dürfen nicht, wie Pipping es fortwäbrend thut, durcheinander geworfen werden. Die Lösung der ersten Frage ist, wie im vorigen Abschnitt hervorgehoben worden ist, unabhängig von derjenigen der zweiten, und ebenso der dritten.

Wenn wir das Hören der Vocale, wie ich sie auffasse, nicht erklären könnten, so müssten wir uns eben bescheiden, da wir die Leistungen des Ohres nur oberflächlich kennen, und am wenigsten kann ein ernster Einwand gegen meine Auffassung daraus entnommen werden, wenn es nicht gelingt, das Hören der Vocale aus einer verbreiteten Hypothese zu erklären.

Aus der schwachen Vertretung des Grundtons in den Vocalklängen hatte ich geschlossen, dass das Hören derselben sehr analog sei demjenigen der Unterbrechungstöne. Das Ohr nimmt die Periodik des Auftretens der anaperiodischen Oscillationen als vorherrschenden Ton wahr, und verleiht diesem Ton den Vocalcharacter, indem ansserdem der Ton der Oscillationen selbst eine Einwirkung ausiibt, auch wenn er fortwährend seine Phase ändert ${ }^{1}$ ).

1) Vgl. dies Archiv Bd. 56. S. $485 \mathrm{ff}$. 
Dass übrigens eine anaperiodisch unbarmonisehe Schwingung auch nach der Helmholtz'schen Tonempfindungslehre das Ohr in ihrem specifischen Character erregen muss, unterliegt gar keinem Zweifel; denn eine solche Schwingung ist, wie ich schon beim Klavierversuch hervorgeboben habe(S.178), stets durch eine Fourier'sche Reihe darstellbar. Dass aber die vorherrschende Empfindung die Note des in der Reile kaum vertretenen Grundtons hat, ist aus der Helmboltz'schen Hypothese nicht erklärbar, wenn man nicht solche Annahmen hinzufügt, wie ich sie zur Erklärung der Intermittenz- und Schwebungstöne gemacht habe.

Pipping macht nun auch gegen meine Auffassung der Intermittenztöne eine Reihe von Einwänden. Gegen die, übrigens nicht zuerst von mir, sondern schon von König vertretene Idee, dass das Obr jede Art ron Periodik als Ton empfindet ${ }^{1}$ ), macht er die höchst befremdende Bemerkung (S. 539): „Hermann hat überseben, dass überall, wo eine Periode von der Schwingungszahl $n$ vorbanden ist, ebenfalls Perioden von den Schwingungszahlen$n / 2, n / 3 \ldots$ da sind. Nach seinem Gesetze müsste jeder Ton von allen seinen Untertönen begleitet sein." Ich kann unmöglich glauben, dass dieser Einwand ernst gemeint ist. Unter Perioden sind doch hier ganz offenbar nur regelmässige Wiederholungen gleichartiger Vorgänge, die in der Zwischenzeit nicht oder anders sind, zu verstehen. Pipping's Einwand ist genau so, als wenn Jemand behauptete, in der Tagesperiodik sei auch die Zweitagsperiodik, die Dreitagsperiodik etc. mit enthalten, und der Mensch, welcher die Tagesperiodik walurnimmt, müsse auch ebenso unmittelbar die Wochenperiode empfinden!

P i p p i n gelbst macht sich die Erklärung der Unterbrechungstöne ungemein leicht. Er stellt nämlich folgenden neuen Lebrsatz auf: wenn wir eine Reihe von Obertönen hören, zu welchen der Grundton fehlt, so bören wir einen Klang von der Note des Grundtons. Nichts kann der Helmboltz'schen Hypothese, welche

1) In meiner ersten Vocalarbeit, Bd. 47. S. 383, 390 habe ich diese Idee ausgesprochen ohne zu wissen, dass dieselbe schon von $\mathrm{K}$ ö $\mathrm{n}$ g geäussert worden ist (vgl. z. B. S. P. Th o m p s on, Proceed. R. Soc. 1890. 13. Juni, p. 14. In einer späteren Arbeit, Bd. 56. S. 493 babe ich Kön ig als Vertreter dieses 'Gedankens ausdrïcklich genannt. Uebrigens haben schon die älteren Physiker, welche die Differenztöne von Schwebungen herleiten, damit stillschweigend dieselbe Annahme gemacht. 
Pipping im Uebrigen auf das Hartnäckigste verficht, mehr widersprechen als dieser Lehrsatz. Dieser Widerspruch gegen eine Hypothese würde ibn freilich für mich keineswegs umstossen; aber die von Pipping beigebrachten Beweise, welche theils anf den bekannten Täuschungen um Octaven, theils auf einem Versuch am Klavier beruhen, scheinen mir bei weitem nicht ausreichend. Wenn übrigens Pipping bemerkt, dass der Grundton in Wirklichkeit selten fehlt, weil er als Differenzton der Obertöne auftritt, so dreht er sich etwas im Kreise; denn die Differenztöne sind eben Schwebungstöne, die es zu erklären gilt; als objective Erscheinungen könnten sie selbst von Jemand, der meine betr. mathematische Beleuchtung ${ }^{1}$ ) gänzlich ignorirt, bei so schwachen Primärtönen wie die Obertöne eines Klangès unmöglich betrachtet werden. Jedenfalls hat $\mathrm{Pipping}$ seiner eigenen Behauptung, dass die Vocalklänge nur nach dem Princip der Fourier'schen Analyse betrachtet werden dürfen, den Boden entzogen.

Bei diesem Anlass polemisirt $\mathrm{P}$ ip p ing auch gegen meine Zahnradversuche, durch welehe ich die Wahrnehmbarkeit eines fortwährend seine Phase wechselnden Tones dargethan habe ${ }^{2}$ ), obwohl diese Frage mit der Vocalfrage nur in ziemlich lockerem Zusammenhang steht. Ich bin genöthigt, auch diesen Thèil der Pippingschèn Arbeit zu beleuchten.

Meine Zahnräder hatten ganz gewöhnliche Zähne, wie jedes S avart'sche Zahnrad; gegen die Zähne wurden beim Rotiren die Ränder dünner Papierdüten, selten Cartonblätter, gehalten. Der Zweek des Savart'schen Rades ist bekanntlich nur; dem Papierblatt $\mathrm{Stösse} \mathrm{in} \mathrm{bestimmten} \mathrm{Intervallen} \mathrm{zu} \mathrm{geben.} \mathrm{Pipping}$ will nun die Zahnradversuche verfeinern, indem er den Scheibenrand in Form von Sinuscurven ausschneidet, und gegen diese Curven Kartenblätter hält; er will also gewissermassen das Princip der Wellensirene verwirklichen und erwartete allen Ernstes, dass das Kartenblatt sich nach dem Gesetze der Curve bewege. Natürlich hat er hier, wie vorauszusehen war, nur Enttäuschungen erlebt; seltsam aber ist, dass er meint, auch ich hätte an meinen Zahnrädern eine ähnliche Erwartung gehabt, denn er fragt (S. 542) nach der Steilheit meiner Curven, während ich gar keine Curven, sondern eben nur Zähne, nicht wesentlich anders gestaltet als in

1) Dies Archiv Bd. 49. S. 502.

2) Dies Archir Bd. 50. S. $489 \mathrm{ff}$. 
meiner schematischen Abbildung, benutzt habe, und die dünnen Papierränder nicht in die Tiefen der "Curven" eingreifen, sondern nur die Zahnspitzen ganz leicht streifen liess. Was Pipping (S. 545) „zeigen wollte", dass nämlich wellenförmige Einschnitte eines Scheibenrandes nicht dem Kartenblatt and der Luft ihr Gesetz mittheilen, hätte man voraussagen können.

Pipping's Zahnradversuche sind aus diesem Grunde mit den meinigen nicht gut vergleichbar; denn grade auf Stösse des Papierblattes und nicht anf Hin- und Herfübrung desselben kann hier viel ankommen. Pipping's Resultate weichen von den meinigen sowohl bei den $A$-Scheiben, d. h. mit Phasenwechsel, wie bei den $B$-Scheiben, d. h. obne Phasenwechsel, ab. Ich gebe an, dass bei beiden Scheiben ausser dem Unterbrechungston, welcher dominirt, der Zahnton gehört wird; Pipping bestätigt dies nur für die $B$-Scheibe, nicht für die $A$-Scheibe; vielmehr hört er bei letzterer statt des Zahntones, der bei ibm 90 Schw. pro Umdr. hat, nur den Ton von 84 und unsicher von $96 \mathrm{Schw}$., welehe der 7. und 8. Theilton des Unterbrechungsklanges sein würden. Ich hatte angegeben, dass der Zahnton der $A$-Scheiben besonders leicht gehört wird, wenn man die Sirene auslaufen lässt, oder so langsam dreht, dass der Unterbrechungston nicht mehr zu Stande kornmt. Hier hat ihn nun auch Pipping offenbar gehört (S. 545), versehanzt sich aber binter der Behauptung, dass beim Auslaufen die Zahnsirene nicht mehr gleichmässig gehe. Woher weiss er denn das? Meine in trefflich gearbeiteten Spitzenlagern gehende Zahnsirene hat eine Schwungscheibe von fast 2 Kilo Gewicht und $141 / 2 \mathrm{~cm}$ Durchmesser; wer diesen Apparat in Bewegung gesehen bat, wird lächeln, dass derselbe beim Auslaufen (die Bewegung nimmt wegen der geringen Reibung und des bohen Trägheitsmoments ungemein langsam ab) durch ein dünnes Papierblatt zu so ungleichmässigem Gange gebracht werden soll, dass man nicht die Töne zweier aufgesetzter Zahnräder durch alternirendes Berühren vergleichen kann. Ausserdem habe ich ausdrücklich angeführt, dass dieselbe Erleichterung für das Hören des Zahntones nicht nur durch das Auslaufen, sondern auch durch langsameren Motorenbetrieb erreicht werden kann.

Ich muss auch nach wiederholten Versuchen meine Angabe vollkommen aufrecht erhalten, und babe mich schon das erste Mal nicht ausschliesslich auf mein eigenes Ohr verlassen, sondern Hrn. 
Dr. Neisser, damals hier Privatdocent und Assistent an der med. Klinik, welcher ein sehr feines musikalisches Gehör besitzt, hinzugezogen. Bei meinen $A$-Scheiben hat der Zahnton wie bei Pipp ing die $7 \frac{1}{2}$-fache Zahl der Unterbrechungen; Pi p ping behauptet nur den 7. und den 8. Oberton des Unterbrechungstones gehört zu haben, welche beide um $1 / 2$ Tonstufe vom Zahnton abliegen. Genau so waren die Verhältnisse bei mir, auch ich strich, wie ich ausdriicklich angegeben habe, die gleichzeitig rotirenden Räder alternirend an, und um sich um einel halben Ton zu irren, dazu gehört schon viel. Bei meiner $C$-Scheibe vollends würde der Irrthum nicht einen halben, sondern einen ganzen Ton betragen baben, und das wird selbst Pipping mir nicht zutrauen.

Trotzdem hielt ich die abweichende Angabe Pipping's für wichtig genug, um die Frage noch einmal mit vollkommneren Hilfsmitteln zu untersuchen. Ich besitze eine Scheibe nach dem Schema $A_{3}$ (dies Archiv Bd. 56. S. 492, und Fig. 6, S. 490), welche 12 Perioden von jo 8 Zähnen mit Phasenwechsel hat. Der Zahnabstand beträgt $4^{\circ}$ (d. h. gleichmässig herumgehend würde die Scheibe 90 Zähne haben), der Periodenabstand nur 20. Diese Scheibe gab bei meinen früheren Versuchen ausser dem Unterbrechungston (oder Phasenwechselton) 12 sebr deutlich den Ton 90 , wie die auf derselben Axe befindliche Normalscheibe von 90 Zähnen. Der Fall ist genau der Pipping'sche; Pipping behauptet aber mit aller Bestimmbeit nur die Töne 84 und (weniger sicher) 96 , d. b. Obertöne des Grundtons 12 gehört zu haben. Strich er nach einander eine Scbeibe mit 96 Zähnen, dann eine Scheibe mit 90 (oder eine dieser Zahl entsprechende $B$-Scheibe), und endlich die Versuchsscheibe $A$ an, so will è dentlich eine absteigende chromatische Tonleiter gehört haben, worin er einen besonders sicheren Beweis sieht, dass die Versuchsscheibe den Ton 84 lieferte. Ich liess nun zwei Normalscheiben mit 84, resp. 96 Zähnen anfertigen, und brachte sie mit der $A$-Scheibe zusammen' auf die Axe (welche zugleich die Normalscheibe 90 wie immer trug). Nie und nimmer hörte ich oder börten andere beim Anstreichen der $A$-Scheibe eine Uebereinstimmung mit der 84- oder 96-Scheibe, sondern immer nur mit der 90-Scheibe, und wenn man successive die 84-, die $A$-, und die 96-Scheibe berührt, so hört man die überzeugendste und zweifelloseste chromatische Tonleiter, die man jeden Augenblick, indem man statt der $A$. Scheibe die 
90-Scheibe anstreicht, mit der reinen durch keinen Intermittenzton gestörten chromatischen Tonleiter vergleichen kann. Ganz besonders überzengend, und für jedes Ohr leicht zum Urtheil führend, wird der Versuch, wenn man geringere Geschwindigkeiten nimmt, oder das Auslaufen der Sirene abwartet, wie ich schon früher hervorhob. Der Unterbrechungston ist nämlich dann viel weniger hervortretend.

Ich hoffe, Pipping wird den Versuch in dieser Form wiederbolen, und sich überzeugen, dass er sich geirrt hat. Da er von vornherein Alles was dem "O hm'schen Gesetz" ${ }^{1}$ ) widerspricht, für unmöglich zu erklären geneigt ist - denn er benutzt Abweichung von diesem Gesetz als Einwand gegen thatsächliche Angaben (S. 541) - so halte ich es nicht für ausgeschlossen, dass er bei diesen Versuchen befangen war, und sich selbst durch die anscheinend auch von ihm erhaltene Bestätigung meiner Angabe an der auslaufenden Sirene nicht tiberzengen liess, sondern einen ganz unzureichenden Ausweg suchte.

Bei den $B$-Scheiben mussten die Perioden etwas ungleich gemacht werden, úm ohne Phasenverschiebung die gleiche Zahl von Unterbrechungen bei gleichem Zahnabstand herzustellen. Hier soll ich nach Pipping mir selber widersprechen, weil ich nicht den Unterbrechungston dev wirklichen, aus je 2 etwas ungleichen Theilen bestehenden Periode, sondern nur den der längeren Theilperiode gehört habe. Welchen Unterbrechungston hier Pipping selbst gehört hat, ist nicht klar zu ersehen, jedenfalls nicht den der ganzen Perioden. In der That drängen sich die Eindrücke der frequenteren Unterbrechungen bedeutend vor, und dass von den beiden entsprechenden, um $1 / 2$ Ton verschiedenen Unterbrechungseindrücken der tiefere dominirt, ist Thatsache. Von einem Widerspruch kann ich hier. Nichts finden, vielmehr liefert die Erscheinung einen Beitrag zu dem noch wenig bekannten Verbalten des Ohres gegenüber Unterbrechungen von wechselndem Intervall. Pipping thut so, als wenn eine Unterbrechung, welche nicht genau in der Mitte zwischen zwei sonst gleichartigen Unterbrechungen liegt, ganz wirkungslos

1) Man kann es allenfalls als "Gesetz" bezeichnen, dass das Ohr aus. den Klängen die Partialtöne heraushört; dass es aber den Schall $n u r$ in der Weise wahrnimmt, dass es ihn harmonisch zerlegt, ist blosse (H el m holtz'sche) Hypothese. 
wäre. Offenbar werden sich diese Unterperioden um so kräftiger geltend machen, je näher sie einander gleich sind; dass Pipping, der sie absichtlich viel ungleicher machte als ich $(2: 3$, bei mir $7: 8$ ), etwas genug $A$ nderes bören m us s te als ich, liegt für jeden Ueberlegenden auf der Hand.

Ich hatte in der Frage der Klangwahrnehmung auch die Baumgarte n'schen sog. "Reflexionstöne" herangezogen ${ }^{1}$ ), welche einen Widerspruch gegen die einfacbe Zerlegungsthèrie darstellen. Pipping hat mich hier missverstanden, wenn er meint, ich hätte diesen Widerspruch nach Pfaudler citirt; ich spreche hier meine e ige ne Meinung aus, und habe Pfaunder und W. Koblrauseh${ }^{2}$ ) nur wegen ihrer Versuche citirt, welche beweisen, dass ein Stosspaar, auch in unregelmässigen Intervallen wiederholt (s. das Schema in meinem Lebrb. d. Physiologie Cap. 12 bei Gehörorgan; 10. Aufl. S. 508, Fig. 94), den dem Paare entsprechenden Ton erzeugt. Die Art wie Pipping mit dem Baumgarten'schen Phänomen umspringt, ist ungewöhnlich oberflächlich. Dem Wasserfallgeräusche künne, meint er, "mit grosser Wahrscheinlichkeit die dem gehörten Tone entsprechende Sinusbewegung als Bestandtheil zngesprochen werden". Wer das schreibt, weiss offenbar nicht, um was es sich eigentlich handelt; warum man grade den von der Reflexionszeit abhängigen Ton $\left.h o ̈ r^{3}\right)$, mag er auch neben unzähligen anderen mit im Geräusch enthalten sein, warum nach Pippi ng's Vorstellungen grade der Ohrresonator dieses Tones vorwiegend angesprochen wird, das gilt es zu erklären. Dass ich selbst später auf Grund meiner Hypothese der Zählzellen eine Erklärung zu geben versucht habe ${ }^{4}$ ), scheint $\mathrm{P}$ ipp ing übrigens entgangen zu sein.

Bekanntlich ist es ausserordentlich schwer, wenn nicht unmöglich, die Vocalformanten unmittelbar, oder mit Resonatoren herauszuhören. Wäre die Verstärkungstheorie richtig, so müsste

1) Dies Archiv Bd. 53. S. 36.

2) Die den Gegenstand nur tangirènde Kohlrausch'sche Mittheilung, die ich meinte, und die P i p ping in Folge eines irrthümlichen Citats von mir nicht finden konnte, steht in W i e d e m a n n's Annalen Bd. 7. S. 335.

3) Vgl. auch Müller-Pouillet's Lehrb. d. Physik. 9. Aufl. v. Pfaunder. Bd. 1. S. 731, 732 .

4) Dies Archiv Bd. 56. S. 496. 
dies im Gegentheil sehr leicht sein. Auch müsste dann ein Vocal zum Vorschein kommen, wenn man neben dem Grundton einen dem Mundton entsprechenden Ton erklingen lässt; nie aber gelingt es anf diesem Wege kiüntlich Vocale zu produciren. Auch die vielfach empfohlene Methode auf eine Pfeife einen Resonator von der Höhe des Vocalformanten zu setzen, wird Niemand befriedigen. Dies Alles spricht entsehieden gegen die Verstärkungstheorie. Die Vocalformanten erklingen, wie die Curven zeigen, stets nur intermittirend, and fast stets mit anaperiodischem Phasenwechsel. Obwohl in der Phase wechselnde Töne vernehmbar sind, treten sie doch sehr zurïck, und wirken mehr modificirend auf den Gesammteindruck, als sie zu einer selbstständigen Tonempfindung Anlass geben. Die wahre Characteristik des Vocals liegt eben in der anaperiodischen Erneuerung. einer Formantschwingung; hierdurch entsteht ein specifischer Gehöreindruck, der sich durch Nachahmung: dieses Vorganges, z. B. durch den Versuch mit der Doppelsirene, künstlich erzeugen lässt. $\mathrm{Ob}$ der Ton des Formanten dabei wirklich zur Wabrnehmung kommt, erseheint fraglich; die Zergliederung des Gesammteindrucks in tonartige Elemente ist kein Postulat, von dessen Erfuillung die Zulässigkeit der vorgetragenen Vorstellung irgendwie abbängen könntẹ.

\section{Schlus sbemerkungen.}

Auf das linguistische Gebiet, besonders auf Versuche, die Vocale nach ihrer Verwandtschaft zu gruppiren, speciell auf die von Pipping versuchte Gruppirung ${ }^{1}$ ), gehe ich vorläufig nicht ein. Dem genannten Autor gegenüber halte ich mich iberbaupt streng in der Defensive, was ich ausdrücklich hervorheben muss, weil schon meine erste ruhige und sachliche Vertheidigung zu meiner grenzenlosen Ueberraschung als ein "heftiger Angriff" bezeichnet worden ist ${ }^{2}$ ).

Ebensowenig bin ich schon jetzt im Stande, auf die Frage der geflïsterten Vocale; und auf die interessante Theorie von Lloy $\mathrm{d}^{3}$ ) einzugeben, da ich hoffe, dass auch von dieser Erscheinung.

1) In der citirten Arbeit in den Acta soc. Fenricae.

2) Zeitschr. f. Biologie Bd. 28. S. 41 .

3) Pho:ietische Studien Bd. 3. S. 251, Bd. 4. S. 37, 183, 275. 
204 L. Ḧ ermann: Weitere Ùntersuchungen über das Wesen der Vocale.

sich mittels des Phonographen analysirbare, Curven gewinnen lassen werden.

Bezuiglich der lauten Vocale sei noch bemerkt, dass die sinnreiche Methode von $\operatorname{Raps}^{1}$ ), welche auf einer Idee von Boltzmann und Töpler beruht, die Möglichkeit in Aussicht stellt, die Sprachlaute ohne jede Benutzung einer vibrirenden Platte oder Membran in Curven darzustellen; dies wäre das Ideal eines Untersuchungsverfahrens. Die bisher auf diesem Wege gewonnenen Curven sind leider für exacte Messung noch nicht scharf genug. Immerhin freue ich mich constatiren za können, dass die Ergebnisse von Raps, welche übrigens nur auf Auszählung oder Proportionalmessung beruhen, in Bezug auf $A$ genau mit den meinigen übereinstimmen, für $U$ nahezu, für $O$ nicht. Andere Vocale sind vom Vf. nicht untersucht.

1) Ann. d. Physik. N. F. Bd. 50. S. 193. Auch als Habilitationsschrift, Berlin 1892. 